\title{
Visual search for dimensionally redundant pop-out targets: Evidence for parallel-coactive processing of dimensions
}

\author{
JOSEPH KRUMMENACHER \\ Rheinisch-Westfälische Technische Hochschule Aachen, Aachen, Germany \\ HERMANN J. MÜLLER \\ Ludwig-Maximilians Universität, Munich, Germany \\ and University of London, London, England \\ and \\ DIETER HELLER \\ Rheinisch-Westfälische Technische Hochschule Aachen, Aachen, Germany
}

\begin{abstract}
In two visual search experiments, the detection of singleton feature targets redundantly defined on multiple dimensions was investigated. Targets differed from the distractors in orientation, color, or both (redundant targets). In Experiment 1, the various target types were presented either in separate blocks or in random order within blocks. Reaction times to redundant targets significantly violated the race model inequality (Miller, 1982), but only when there was constancy of the target-defining dimension(s) within trial blocks. In Experiment 2, there was dimensional variability within blocks. Consistent with Experiment 1, constancy of the target-defining dimension(s), but this time across successive trials (rather than within blocks), was critical for observing violations of the race model inequality. These results provide evidence for parallel-coactiveprocessing of multiple dimensions, consistent with the dimension-weighting account of Müller, Heller, and Ziegler (1995).
\end{abstract}

In many workplaces, the operator's task involves frequent and efficient scanning of displays with high information density, and fast detection of and reaction to target objects may be vital (e.g., Wickens, 1992). Therefore, target objects that need to be inspected urgently should be made highly salient in order to summon attention. There are several means of manipulating the saliency of display objects. One efficient means is to create feature contrast between the target and the background (distractor) objects (e.g., Nothdurft, 1991, 1993; Wolfe, 1994). The finding that objects that differ from a more or less homogeneous background in a salient feature are detected rapidly, and independently of the number of distractors, has been referred to as pop-out (e.g., Treisman, 1988). Most theories of visual search assume that feature contrast is computed by early, preattentive mechanisms (see below).

The present study investigated whether the combination of two such contrasts within a single target stimulus would permit faster and more accurate task performance,

This work was supported by Deutsche Forschungsgemeinschaft Grants HE 1132/5-1 and Schr 375/8-1. We thank Asher Cohen and one anonymous reviewer for their valuable comments on a draft of this article. Correspondence concerning this article should be addressed to J. Krummenacher, Institut für Psychologie, Rheinisch-Westfälische Technische Hochschule Aachen, Jägerstr. 17/19, D-52056 Aachen, Germany (e-mail: joseph.krummenacher@ post.rwth-aachen.de). in comparison with the detection of targets defined by feature contrast in one dimension only. Surprisingly, there is as yet relatively little systematic knowledge concerning the effect of combining two (or more) feature dimensions in such a redundant way on visual search performance.

The most detailed investigation of redundant target processing to date has been presented by Mordkoff and his colleagues (e.g., Mordkoff, Miller, \& Roch, 1996; Mordkoff \& Yantis, 1993; Mordkoff, Yantis, \& Egeth, 1990), who have also provided very thorough analyses of methodological problems involved in distinguishing between various models designed to explain redundancy gains (see below). However, the number of objects in their displays was generally small (e.g., one or two in the study of Mordkoff \& Yantis, 1993), and the task required target identification rather than mere detection (e.g., identification of the target-defining features, involving matching of the actual stimulus presented with memorized information defining possible targets, in Experiment 1 of Mordkoff \& Yantis, 1993). Thus, it cannot be taken for granted that their findings generalize to the detection of any odd-oneout object in a search display exhibiting feature contrast in one or two dimensions to a large number of densely spaced, homogeneous background objects. The present experiments were designed to address this issue, with the particular feature dimensions examined being color and orientation (i.e., form). The following sections will review relevant theories of visual search in order to derive predic- 
tions concerning the effects of redundant target definition on detection reaction times (RTs).

\section{VISUAL SEARCH FOR ODD-ONE-OUT FEATURE TARGETS}

It is well established that targets that differ from distractors in certain single salient features can be rapidly discerned irrespective of the number of items in the display. Visual features that support set-size-independent search (including orientation, size, color, stereo depth, and motion) are generally assumed to be registered in parallel across the visual field. There are various accounts of how salient feature differences in the field may be detected. One influential account is Guided Search (GS; Cave \& Wolfe, 1990; Wolfe, 1994). GS assumes that the visual field is initially represented, in parallel, as a set of basic stimulus attributes in different dimensional modules (such as color, orientation, etc.). Each module computes saliency signals for all stimulus locations, indicating the feature contrast of one particular item relative to the various other items represented within the same module. The more dissimilar an item is, as compared with the others, the greater its saliency. Maps of saliency signals are computed in parallel in all modules, and then these signals are summed onto a master map of activations. The activity on the master map guides focal attention, the most active location being sampled with priority (see also Koch \& Ullman, 1985). Focal attention gates the passage of information to higher stages of processing (visual object recognition and response systems). Thus, any odd-one-out feature target will generate a strong contrast signal within its own dimension. Even given some variability owing to noise, the target's saliency signal on the master map should always be larger than those of distractor items, and attention should always be deployed first to its location.

However, recent work (Found \& Müller, 1996; Müller, Heller, \& Ziegler, 1995; Treisman, 1988) has demonstrated that bottom-up models, such as early versions of GS, are, in a crucial respect, incomplete as an account of singleton feature search-in particular, when the dimension defining the target is uncertain on a trial (i.e., variable across trials). Dimensional variability produces two related effects: a cross-dimension search cost and a dimension-specific intertrial transition effect. A crossdimension search cost consists of an increase in search RTs when the target dimension is unpredictable on a trial (cross-dimension search), relative to when the target feature is unpredictable (within-dimension search), without there being an RT cost for within-dimension search relative to a control condition in which both the target dimension and the feature value are certain. There is a dimension-specific intertrial transition effect when detection of a target on a given trial is delayed when the target dimension changes from the preceding to the current trial, but there is no or only little delay when the target feature value changes (with the dimension remaining constant). ${ }^{1}$ These effects are inconsistent with the assump- tion that saliency signals from relevant dimensions are integrated by the master map units in a parallel and equally weighted fashion.

Müller and his colleagues took the cross-dimension cost and intertrial facilitation observed in their experiments to argue for a dimension-weighting account of visual search for feature targets. Similar to GS, for a feature target to generate fast parallel search requires that it rapidly attract focal attention. Focal attention operates on a master map of integrated (summed) dimensionspecific saliency signals. However, unlike early versions of GS, dimension-specific saliency information is attentionally weighted as it is transmitted to the master map of activations. Sufficient weight must be assigned to the target dimension for the target's saliency signal at the master map level to exceed the response threshold. In the within-dimension conditions described above, the target dimension was always known and, thus, weighted in advance, permitting rapid search (i.e., weights may be assigned according to the known likelihood of a target appearing in a particular dimension). However, in the cross-dimension conditions (in the absence of knowledge of the likely target dimension), the search involved a timeconsuming weight-shifting process to determine the target's dimension and amplify its activity at the master map level. The weight pattern established in this process persists into the next trial, producing a dimension-specific RT advantage for a target defined within the same dimension as the preceding target. ${ }^{2}$

In summary, recent work on visual search for singleton feature targets across dimensions suggests that target detection requires some form of checking of the relevant dimensions. This checking could, in principle, proceed either serially or in parallel. In terms of the dimensionweighting account, does the weighting process operate in parallel in multiple dimensions or serially in only one dimension at a time? 3

\section{Coactive Processing of Redundantly Defined Feature Targets?}

Although some theorists have advocated serial processing of dimensions (e.g., Grossberg, Mingolla, \& Ross, 1994; Treisman, 1988), ${ }^{4}$ other evidence (e.g., Müller et al., 1995) points toward parallel processing. Note that the dimension-weighting account as such makes no prediction as to whether singleton feature search across dimensions is serial in nature or parallel and, if the latter, whether a parallel-race (or separate-activation) model (a parallel race of independent redundant target signals to activate the detection mechanism) or, alternatively, a parallel-coactivation model (coactivation of the detection mechanism by redundant target signals) is true. The same applies to alternative accounts of the cross-dimension search cost and dimension-specific intertrial transition effects described above (e.g., Cohen \& Magen, 1999). Thus, the issue of serial versus parallel processing of dimensions is an empirical one. One means of resolving this issue conclusively is by examining visual search for singleton feature targets redundantly defined on multiple 
dimensions - more specifically, by adapting the redundanttarget search paradigm (e.g., Mordkoff \& Yantis, 1993; Mordkoff et al., 1990) to cross-dimension search, permitting Miller's (1982) race model inequality to be tested.

Normally, in redundant-target search, there can be one or two targets in the display (on present trials). Serial search models predict a redundancy gain so that mean RTs should be faster when there are two targets than when there is only one, simply because one of two targets has a higher chance of being encountered early in the search than a single target. However, when the entire distributions of RTs are analyzed (rather than just mean RTs), a form of redundancy gain may be revealed that is inconsistent with any strictly serial or parallel-race model. Miller (1982) demonstrated that all models that assume that each target produces an independent, separate activation must satisfy the following race model inequality: $P(\mathrm{RT}<$ $\left.t / \mathrm{T}_{1} \& \mathrm{~T}_{2}\right) \leq P\left(\mathrm{RT}<t / \mathrm{T}_{1}\right)+P\left(\mathrm{RT}<t / \mathrm{T}_{2}\right)$, where $t$ is the time since display onset and $\mathrm{T}_{1}$ and $\mathrm{T}_{2}$ are Targets 1 and 2. Importantly, this inequality entails that the fastest RTs to displays with redundant targets be no faster than RTs to displays with single targets; however, fast RTs may occur more often with redundant targets. Violations of this inequality constitute evidence against both serial and parallel-independent processing and in favor of parallelcoactive processing.

Applied to cross-dimension search, the question of serial versus parallel search of dimensions can be resolved by varying the number of dimensions on which a single target is defined (instead of varying the number of targets in a display) - for example, color only or orientation only (singly defined targets) or color and orientation simultaneously (redundantly defined target). It can then be examined whether only one dimension (dimension-specific saliency signal) at a time can activate a response-relevant (e.g., master map) representation or whether there is coactivation from multiple dimensions. If the fastest RTs to redundantly defined targets are found to be faster than the fastest RTs to singly defined targets, this would violate the race model inequality, constituting strong evidence for parallel-coactive processing of dimensions.

To illustrate, if relevant dimensions are checked serially, a dually (redundantly) defined target should be detected only as quickly as the fastest singly defined target. Thus, for instance, if color is searched first as a matter of strategy (e.g., on the basis of the experience that popout occurs fastest for this dimension), a target defined by color only would be detected as quickly as a target redundantly defined by both color and orientation - in terms of both the fastest RTs and the average RTs. If relevant dimensions are searched randomly, each singly defined target would have a lesser chance of being encountered early during the search than would a redundant target. This would slow the average detection RTs for both single targets, by reducing the probability of fast detection RTs and increasing that of slow RTs. Nevertheless, the fastest RTs would be as fast as those for redundant targets-albeit, less frequent. In contrast, if the relevant dimensions are processed in parallel and coactively, the fastest redundant-target RTs can be faster than the fastest single-target RTs, because the feature contrast signals in both dimensions would combine to raise the target's activation on the master map above the value achieved by a single-dimensional saliency signal. This should be relatively independent of the speed with which each singledimensional saliency signal becomes available and independent of expectation-based strategies (to assign priority to one, rather than the other, dimension).

Evidence for parallel-coactive processing of color and form has been provided by Mordkoff and Yantis (1993). In their Experiment 1, participants were presented with a single central letter (of size $1.40^{\circ} \times 0.89^{\circ}$ ), an $\mathrm{X}, \mathrm{O}$, or I, colored green, cyan, or purple. Participants had to produce a go response (go/no-go task) whenever the stimulus was an $x$ (irrespective of its color) or green (irrespective of its shape). Redundantly defined targets, green Xs, generated RTs that significantly violated the race model inequality. However, as was pointed out above, it is not clear whether this finding extends to tasks requiring simple detection of any odd-one-out stimulus in a large search array.

\section{Constancy/Variability of Target Definition Across Trials}

One further issue related to the question of serial versus parallel-coactive processing of dimensions concerns how RT redundancy gains are influenced by the constancy or variability, respectively, of target definition across trials. Serial checking of dimensions would imply that a significant benefit of redundant target definition exists only in cases of dimensional variability, when the observer cannot tell in advance whether a target is singly defined in one dimension or redundantly in two dimensions, and only for the slower range of the RT distribution (this assumes that search priority is not assigned consistently to one dimension). The benefit could, therefore, disappear if the task conditions promote the development of correct expectations as to the particular dimension in which an upcoming target will be defined-in particular, under conditions of dimensional constancy. In contrast, if dimensions are processed in a parallel-coactive manner, redundantly defined targets would be detected faster in both constant- and variable-dimension conditions, regardless of the extent of an observer's knowledge-based expectations, and over the whole range of the RT distribution. To examine these predictions, the present study varied the constancy/variability of target definition by presenting singly and redundantly defined targets either in separate trial blocks (constant-dimension condition) or in randomized order within blocks (variable-dimension conditions).

The blocking of the target-defining dimension(s) in experiments testing for violations of the race model inequality raises a methodological problem. Under constantdimension conditions, observers could lower their decision criteria during (blocks of) redundantly defined targets, relative to (blocks of) singly defined targets (e.g., see Grice, 1968, for the argument that the criterion value 
may be determined by, or "adapt" to, constant signal intensity), which could give rise to artifactual RT redundancy gains that violate the race model inequality. However, it should be possible to detect such confounding criterion adjustments by one or two markers. The first is that, in a search task requiring target-present versus target-absent responses, lowered decision criteria should lead not only to faster RTs, but also to higher error (in particular, false alarm) rates. This marker is weak, however, since there are situations in which increased (i.e., redundant-target) signal strength combined with lowered decision criteria could lead to lower false alarm rates for redundantly defined targets than for singly defined targets. A second marker would be the finding of (artifactual) violations of the race model inequality not only for target-present RTs, but also for target-absent RTs. This assumes that negative responses are produced when a response deadline is exceeded, which is set in accordance with some implicit knowledge of the average time it takes to detect the target and the variance of the positive responses (see Chun \& Wolfe, 1996, for a discussion of how negative responses may be made in visual search). That is, a certain time may be added to the positive-decision criterion so that the risk of missing target signals at the slow end of the positive-response distribution is kept at an acceptable level. Furthermore, assuming that strong signals have smaller variance (see Zohary, Shadlen, \& Newsome, 1994), the added time could be even shorter than it is when signals are weak. Thus, given that criterion adjustments to signal strength hold for negative as well as for positive decisions, target-absent RTs should also violate the race model inequality, even though there are no feature contrast signals that could coactivate a response. Thus, it remains an empirical issue whether any RT redundancy gains observed under constant-dimension conditions are confounded by criterion adjustments. Finally, it is possible to explore the effects of dimensional constancy under conditions in which singly and redundantly defined targets vary randomly across trials - that is, when the stimulus contingencies are biased against repetition of redundant targets on consecutive trials. In summary, there are no a priori (methodological) reasons not to explore the effects of dimensional constancy on redundant target processing, whereas there are good theoretical reasons to investigate them. Nevertheless, extreme caution is indicated in interpreting violations of the race model inequality under constant-dimension conditions.

\section{EXPERIMENT 1}

In Experiment 1, the effects of redundant target coding on mean RTs, error rates, and RT distributions were examined by using a conventional odd-one-out feature search task. Observers were presented with (25 green) circles, demarcated by square surround frames (similar to indicators used in modern cockpit displays; see Figure 1). The target circle was singled out from the irrelevant distractor dials by having a surround frame that was unique in color (red square among green squares), in orientation (green diamond among green squares), or in both color and orientation (red diamond among green squares), so that it could be tested whether targets defined in both dimensions would produce redundancy gains, relative to targets defined in one dimension only. The observers had simply to discern the presence of a target outline frame.

Redundancy gains were examined under both a constantdimension condition, with one target type per trial block (similar to Monk's, 1976, study), and a variable-dimension condition, with the various target types presented in random order within blocks. From a number of applied and basic studies (e.g., Monk, 1976; Müller et al., 1995; Treisman, 1988), uncertainty as to the target-defining dimension(s) would be expected to increase RTs in general.
A.

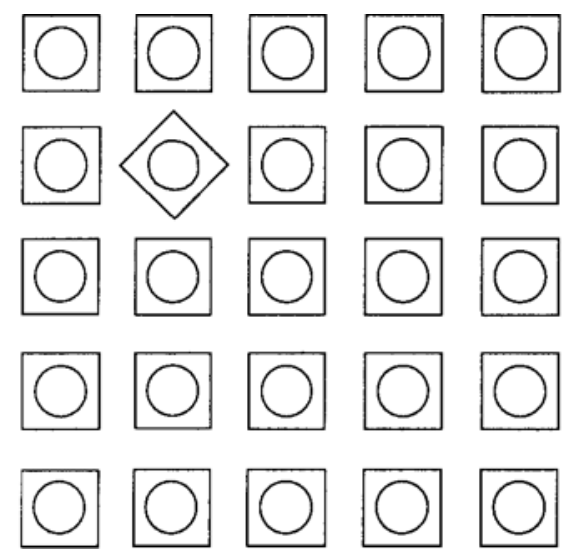

B.

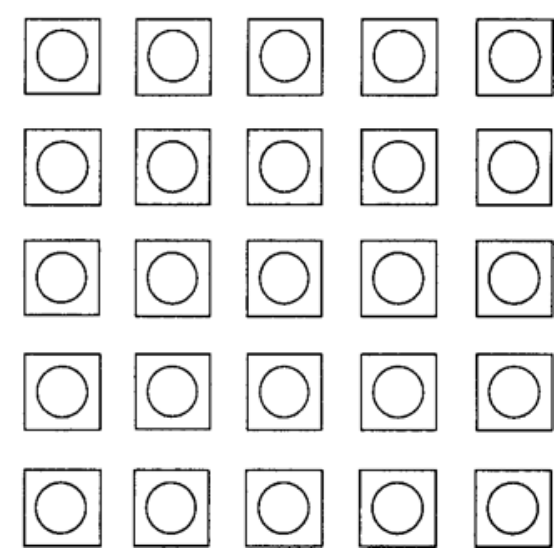

Figure 1. Stimuli presented in Experiment 1. Panels A and B illustrate target-present and target-absent displays, respectively. The target in panel $A$ is orientation defined, indicated by a diamond frame. 
Furthermore, some applied research (Backs \& Walrath, 1995; Christ, 1975) would suggest that a benefit of redundant (color) coding may be obtained only if observers are aware of the constancy of target definition. In contrast, as was discussed above, a serial model of dimensional processing might predict an interaction in the opposite direction - that is, a redundancy gain would be expected in the variable-dimension condition, but not in the constant-dimension, condition.

A third issue examined in Experiment 1 concerned the effects of the relative efficiency of color and orientation coding on redundant-target detection. In many studies of feature search, color-defined targets are detected faster than orientation-defined targets, even under blocked conditions (e.g., Krummenacher, Müller, \& Heller, in press; see also Theeuwes, 1991, 1992), suggesting that color saliency is derived more rapidly than orientation saliency. Consistent with this, Moutoussis and Zeki (1997) have recently argued that noncolor (i.e., motion) signals must precede color signals by a considerable time $(70-80 \mathrm{msec})$ to be integrated, or "bound" with color information. Suppose that there is a stage at which saliency signals from multiple dimensions combine to coactivate a detection device. This raises the question of whether this stage is sensitive to the temporal order of color and noncolor signals along the lines suggested by Moutoussis and Zeki (and whether it is the same stage as the visual-binding stage studied by Moutoussis and Zeki). If so, the mean RT redundancy gains and violations of the race model inequality should be more marked when orientation saliency is derived faster than color saliency. To investigate this issue, Experiment 1 included conditions in which orientation targets were processed more efficiently than color targets and vice versa.

\section{Method}

Participants. Ten observers participated in Experiment 1. Their ages ranged between 17 and 33 years (mean, 27.7 years); 4 were female, and 6 were male. All the observers had normal vision (including color vision).

Apparatus. The observers viewed the display from a distance of $57 \mathrm{~cm}$, with eye-screen distance maintained through the use of a chinrest. The experiment was conducted in a darkened room with dim background lighting, to prevent screen reflections. A Sony Trinitron Multiscan 17 sfII color monitor was used (frame rate, $100 \mathrm{~Hz}$ ), controlled by a Dell P75 computer equipped with an ELSA WINNER 2000 AVI graphics card. The observers responded by pressing the left or right mouse button with the index finger of the left or right hand, respectively. The mouse track ball was removed to increase the accuracy of the timing (Segalowitz \& Graves, 1990). RTs and error rates were recorded by the computer. A computergenerated error feedback signal (bleep) was given when an erroneous response was made.

Stimuli and timing. The display consisted of a matrix of $5 \times 5$ stimuli (see Figure 1). The display elements were green (VGA color $0,248,0)$ circles framed by green squares for the distractor stimuli. The target frame was either differently colored (red [VGA color $255,0,0$ ] or turquoise [VGA color $0,235,40]$ ), differently oriented (diamond orientation [i.e., square tilted by $45^{\circ}$ ]), or both (see below). The green, red, and turquoise colors were matched for luminance $\left(3.4 \mathrm{~cd} / \mathrm{m}^{2}\right)$. The inner circles of the stimuli were empty. The size of the stimulus frames was $1.5^{\circ} \times 1.5^{\circ}$ of visual angle (frame), that of the inner circles was $1.2^{\circ}$ (diameter), and the distance between the stimulus frames was $1^{\circ}$. The screen background was black. The target was always presented at one (randomly selected) location of the inner $3 \times 3$ display matrix to avoid edge effects - that is, slower detection of target items presented at the edge of a display. The observers were not informed of this. The target location was varied randomly within this inner matrix.

There were two (target) color conditions. In each condition, the target was marked by a red or a turquoise square frame (color target), a green diamond frame, or a red or a turquoise diamond frame (redundant condition). Pilot work conducted prior to Experiment 1 had shown that detection of the red color target was slightly more rapid than detection of the $\left(45^{\circ}\right)$ orientation target, whereas the detection of the turquoise color target was somewhat less rapid (RT differences $<20 \mathrm{msec}$ ).

A trial started with the simultaneous onset of all 25 inner circles, which were all green, followed after $500 \mathrm{msec}$ by the appearance of the outer frames. This display remained on the screen until the observer responded (or, after an erroneous response, until after the error feedback signal). The intertrial interval was $1,050 \mathrm{msec}$.

Design and Procedure. All the observers performed both (target) color conditions (red or turquoise), with the target-defining dimension either constant throughout a block of trials or variable (dimensional variability, constant or variable). Targets were color defined, orientation defined, or redundantly defined (target dimension, color, orientation, or redundant). The task required target detection - that is, a decision as to whether or not the display contained an odd-framed target object. The observers were instructed to react as quickly and as accurately as possible.

All the observers participated in the four experimental (color $\times$ dimensional variability) conditions. Half of the observers performed the constant (dimension) conditions first, and half the variable conditions. Within each constant and variable condition, the observers performed the two color conditions (red or turquoise) in counterbalanced order. Each observer was tested on 2 different days, each day devoted to one or the other dimensional variability condition.

Immediately prior to an experimental (color $\times$ dimensional variability) condition, the observers were familiarized with the task in one practice block of 60 trials. In constant-dimension conditions, the dimension defining the target during a given block was illustrated to the observers for $30 \mathrm{sec}$ before they could initiate the first trial, by displaying the stimulus matrix containing the appropriate target (red/turquoise square, green diamond, or red/turquoise diamond). The target-defining dimension(s) (color, orientation, or redundant) changed from block to block. In variable-dimension conditions, all three possible target types were shown to the observers at the very beginning of each condition, informing them that the target type was not fixed.

Each of the four experimental conditions consisted of nine blocks of 63 trials (not counting practice) and lasted for about $30 \mathrm{~min}$. Each block comprised three unrecorded warm-up trials, followed by 60 experimental trials ( 30 target-absent trials and 10 trials each with a color target, an orientation target, and a redundant target) plus a number of trials equal to the number of errors made, since error trials were repeated at the end of each block. Between blocks, the observers were free to take a short break.

\section{Results and Discussion}

The RT results are presented in three consecutive sections examining the mean redundancy gains, intertrial effects (variable-dimension condition), and RT distributions. The error data are presented subsequently. To eliminate anticipatory or delayed reactions, RTs at the extreme ends of the distribution (shorter than $100 \mathrm{msec}^{5}$ and longer than $1,000 \mathrm{msec}$; less than $1 \%$ of the data) were excluded from the analyses. 
Table 1

Mean Correct Reaction Times (RTs, in Milliseconds) to Color-Defined, Orientation-Defined, and Redundantly Defined Targets, Separately for the Red- and Turquoise-Color and the Constant- and Variable-Dimension Conditions in Experiment 1

\begin{tabular}{|c|c|c|c|c|c|}
\hline \multirow[b]{2}{*}{ Condition } & \multicolumn{3}{|c|}{ Target } & \multirow[b]{2}{*}{ Gain ${ }^{B} \& C$} & \multirow[b]{2}{*}{ Gain M\&L } \\
\hline & Color & Orientation & Redundant & & \\
\hline \multicolumn{6}{|c|}{ Target Present } \\
\hline \multicolumn{6}{|l|}{ Constant } \\
\hline Red & 390.6 & 401.8 & 366.3 & 24.3 & 19.9 \\
\hline Turquoise & 418.0 & 404.2 & 384.2 & 20.0 & 17.4 \\
\hline \multicolumn{6}{|l|}{ Variable } \\
\hline Red & 402.4 & 425.2 & 378.7 & 23.7 & 18.8 \\
\hline Turquoise & 438.6 & 422.4 & 399.5 & 22.9 & 23.3 \\
\hline \multicolumn{6}{|c|}{ Target Absent } \\
\hline \multicolumn{6}{|l|}{ Constant } \\
\hline Red & 413.3 & 423.4 & 399.9 & 13.4 & 7.7 \\
\hline Turquoise & 438.8 & 427.3 & 426.3 & 1.0 & 2.2 \\
\hline \multicolumn{6}{|l|}{ Variable } \\
\hline Red & 434.2 & 434.2 & 434.2 & - & - \\
\hline Turquoise & 442.3 & 442.3 & 442.3 & - & - \\
\hline
\end{tabular}

Note-Gain ${ }^{\mathrm{B} \& \mathrm{C}}$, RT redundancy gain relative to the, on average, fastest single-dimension target condition (Biederman \& Checkosky, 1970); Gain ${ }^{\mathrm{M} \& \mathrm{~L}}$, RT redundancy gain computed according to Miller and Lopes (1988).

Mean RT redundancy gain. Table 1 (upper half) presents the mean correct positive RTs (in milliseconds) for color-defined, orientation-defined, and redundantly defined targets, as well as the mean redundancy gain relative to the fastest single-dimension condition, separately for each dimensional variability (constant or variable) and color (red or turquoise) condition. As can be seen, there were mean RT redundancy gains of the order of 20-25 msec in all the conditions.

A repeated measures analysis of variance (ANOVA), with main terms for dimensional variability (constant or variable), color (red or turquoise), and target dimension (color, orientation, or redundant), revealed the main effects of variability $\left[F(1,9)=15.95, M S_{\mathrm{e}}=540.8, p<\right.$ $.005]$, and dimension $\left[F(1.8,16.2)=55.24, M S_{\mathrm{e}}=252.0\right.$, $p<.001$; Huynh-Feldt corrected degrees of freedom] to be significant. Furthermore, the color $\times$ dimension interaction was significant $\left[F(1.2,10.6)=5.65, M S_{\mathrm{e}}=782.0\right.$, $p<.05$; Huynh-Feldt corrected degrees of freedom]. No other effects were revealed to be significant.

Constant-dimension RTs were somewhat faster overall than variable-dimension RTs (394.2 vs. $411.1 \mathrm{msec}$ ). More important, redundantly defined targets were responded to faster than singly defined targets $(382.2,412.4$, and $413.4 \mathrm{msec}$ for redundant, color, and orientation targets, respectively). The color $\times$ dimension interaction was due to red-color targets being responded to faster than orientation targets ( $396.5 \mathrm{vs} .413 .5 \mathrm{msec}$ ), whereas turquoise-color targets were responded to slower than orientation targets (428.3 vs. $413.3 \mathrm{msec}$ ). Despite these color-orientation differences, the mean redundancy gains relative to the, on average, fastest singly defined targets were equivalent ( 24.0 vs. $21.5 \mathrm{msec})$, and they did not differ between the constant- and the variable-dimension conditions ( 22.1 vs. $23.3 \mathrm{msec}$ ).
As a test of mean RT redundancy gains, the above analysis may be referred to as a fixed-favored dimension test (see Biederman \& Checkosky, 1970), because it implicitly assumes that all the observers preferred the same one of the two single target-defining dimensions. However, this assumption is incorrect: Some observers did favor the other dimension, leading to an overestimation of the mean RT redundancy gain relative to the (for each observer) fastest single-dimension condition. (This does not render the above ANOVA-as an assessment of the average efficiencies of the various color and orientation targets in determining target detection-obsolete.) On the other hand, using only the faster of the two singledimension RTs for a given observer may lead to an underestimation of the mean RT redundancy gain, owing to random variation in mean RTs in the two single-dimension conditions. To deal with these problems, Miller and Lopes (1988) devised an alternative procedure. Their test involves the comparison of the two single-dimension conditions for each observer separately. When the two conditions differ (using a liberal criterion of $\alpha=.10$ ), the faster mean RT is retained as the conservative estimate of single-dimension mean RT; when the two conditions do not differ, the overall mean from both single-dimension conditions is retained. These estimates are then compared across all observers with their redundant-target RTs. The mean RT redundancy gains computed according to the Miller-Lopes procedure (Gain ${ }^{\mathrm{M} \& \mathrm{~L}}$ ) are presented in the right-most column of Table 1, alongside those calculated according to the Biederman-Checkosky procedure $\left(\right.$ Gain $\left.^{\mathrm{B} \& \mathrm{C}}\right)$. The Miller-Lopes gains were examined with a repeated measures ANOVA, with main terms for dimensional variability (constant or variable), color (red or turquoise), and target dimension (single $\mathrm{M}^{\mathrm{ML}}$, or redundant, where single ${ }^{\mathrm{M} \& \mathrm{~L}}$ is the best single-dimension target RT estimate based on the Miller-Lopes procedure). This ANOVA revealed the main effect of dimension $[F(1.2,14.5)$ $=66.86, M S_{\mathrm{e}}=206.5, p<.001 ;$ Huynh-Feldt corrected degrees of freedom] to be significant, whereas there were no significant interactions involving dimension. In other words, the Miller-Lopes gains were reliable and did not differ significantly between the red and the turquoise colors (19.4 vs. $20.4 \mathrm{msec})$ and between the constant- and the variable-dimension conditions (18.7 vs. $21.1 \mathrm{msec}$ ).

In summary, there were significant mean RT redundancy gains that were independent of the particular dimension (color or orientation) that was processed fastest. This suggests that the redundancy gains arise at a stage at which no dimension is special (in contrast to the perceptual binding stage studied by Moutoussis and Zeki, 1997, at which feature binding may be delayed until color information becomes available). Furthermore, there were significant mean RT redundancy gains not only under variable-dimension, but also under constant-dimension conditions. This finding argues against serial models of dimensional processing. Under blocked conditions, the serial model would predict that the RT to a redundantly defined target would be equal to or slower than that to the faster one of the two singly defined targets (equal if the 
observer always checks the "fastest" dimension first, slower if otherwise). Thus, the finding of a mean RT redundancy gain in the constant-dimension condition argues against serial processing and in favor of some form of parallel processing of dimensions (race of independent dimensional saliency signals or coactive processing).

Similar to the positive RTs, negative RTs (Table 1, lower half) were overall slower in the variable-dimension condition than in the constant-dimension condition [438.3 vs. $\left.421.5 \mathrm{msec} ; F(1,9)=10.29, M S_{\mathrm{e}}=272.6, p<.025\right]$. Furthermore, in the constant-dimension condition, negative RTs showed the same pattern as positive RTs: They were fastest in the redundant-target condition, whether the target-defining color was red or turquoise, and the relative response speed in the color and orientation target conditions depended on the target-defining color (red, color < orientation; turquoise, orientation $<$ color). An ANOVA of the negative RTs in the constant-dimension condition, with main terms for color (red or turquoise) and target dimension (color, orientation, or redundant), revealed the main effect of dimension to be significant $\left[F(1.6,14.4)=4.99, M S_{\mathrm{e}}=265.1, p<.05 ;\right.$ Huynh-Feldt corrected degrees of freedom]. (Further analyses revealed both the Biederman-Checkosky [1970] redundancy gains $\left[F(1,9)=21.3, M S_{\mathrm{e}}=74.3, p<.001\right]$ and the MillerLopes [1988] redundancy gains $\left[F(1,9)=5.92, M S_{\mathrm{e}}=\right.$ $100.9, p<.05]$ to be significant.) This pattern of effects suggests that, in the constant-dimension condition, negative RTs are determined by the speed with which the presence of a target can be discerned; in the variabledimension condition, they are determined by the speed with which the slowest of the target alternatives is detected. In other words, negative responses are given if an adjustable response deadline is exceeded, which is set in accordance with how rapidly a constant target or, respectively, the slowest of variable targets can be detected (so as to keep the rate of target misses at an acceptable level). This is consistent with the reasoning outlined in the introduction (see the Constancy/Variability of Target Definition Across Trials section).

Intertrial effects (variable-dimension condition). Table 2 presents the mean correct RTs to color-defined and orientation-defined targets on a given trial $(n)$, de-

Table 2

Mean Correct Reaction Times (in Milliseconds) to Color-Defined $(C)$ and Orientation-Defined $(O)$ Targets on Trial $n$, Depending on Whether the Target on Trial $n-1$ Was Defined in the Same Dimension, in Both Dimensions (Redundant, R), or in a Different Dimension, Separately for the Red-and the Turquoise-Color Conditions in Experiment 1

\begin{tabular}{lccc}
\hline & \multicolumn{3}{c}{ Target $n-1$} \\
\cline { 2 - 4 } Condition & Same & Redundant & Different \\
\hline Color target $n$ & $\mathrm{C} C$ & $\mathrm{R} C$ & $\mathrm{O} C$ \\
\cline { 2 - 4 } Red & 391.3 & 400.7 & 404.7 \\
Turquoise & 421.2 & 433.1 & 444.1 \\
Orientation target $n$ & $\mathrm{O} O$ & $\mathrm{R} O$ & $\mathrm{C} O$ \\
\cline { 2 - 4 } Red & 408.4 & 429.4 & 423.6 \\
Turquoise & 407.1 & 429.9 & 429.9 \\
\hline
\end{tabular}

Table 3

Mean Correct Reaction Times (in Milliseconds) to Redundantly Defined Targets on Trial $n(R)$, Depending on Whether the Target on Trial $n-1$ Was Defined in the Orientation $(O)$ or the Color $(C)$ Dimension or in

Both Dimensions (Redundant, R), Separately for the Red- and the Turquoise-Color Conditions in Experiment 1

\begin{tabular}{lccc}
\hline & \multicolumn{3}{c}{ Target $n-1$} \\
\cline { 2 - 4 } Condition & $\begin{array}{c}\text { Orientation } \\
(\mathrm{O} R)\end{array}$ & $\begin{array}{c}\text { Color } \\
(\mathrm{C} R)\end{array}$ & $\begin{array}{c}\text { Redundant } \\
(\mathrm{R} R)\end{array}$ \\
\hline Red & 377.0 & 368.8 & 365.5 \\
Turquoise & 402.5 & 392.2 & 395.5 \\
\hline
\end{tabular}

pendent on whether the target on the preceding trial $(n-$ 1) was singly defined in the same dimension, redundantly defined in both dimensions (redundant), or singly defined in a different dimension, separately for the red-and the turquoise-color conditions. A repeated measures ANOVA, with main terms for color (red or turquoise), target $n$ (target dimension on trial $n$, color or orientation), and target $n-1$ (target dimension on trial $n-1$, same, redundant, or different) revealed the main effect of target $n-1$ to be significant $\left[F(2,18)=4.57, M S_{\mathrm{e}}=897.5, p<\right.$ .025]: RTs were significantly faster when the preceding target was defined within the same dimension than when it was redundantly defined or defined within the other dimensions (407.0 vs. 423.3 and $425.6 \mathrm{msec}$, respectively).

Table 3 presents the mean correct RTs to redundantly defined targets on a given trial $(n)$, depending on whether the preceding target $(n-1)$ was defined in the orientation or the color dimension or in both dimensions (redundant), separately for the red-and the turquoise-color conditions. A repeated measures ANOVA, with main terms for color condition and target $n-1$ revealed no significant effects.

One further comparison of interest concerns that between the second of two consecutive color-color (CC) targets, orientation-orientation $(\mathrm{O} O)$ targets, and redundantredundant $(\mathrm{R} R)$ targets: $\mathrm{R} R$ targets were responded to significantly faster than $\mathrm{C} C$ and $\mathrm{O} O$ targets [380.5 vs. 406.3 and $407.8 \mathrm{msec}$, respectively; $F(2,18)=15.22$, $\left.M S_{\mathrm{e}}=308.3, p<.001\right]$.

In summary, the dimension of the target on trial $n-1$ significantly influenced responses to the target on trial $n$. There were costs in responding to singly defined targets when the preceding target was defined in a different dimension or when it was redundantly defined. The faster RTs for same-dimension targets on consecutive trials relative to different-dimension targets are consistent with Found and Müller (1996), who attributed this effect to the persistence of the pattern of dimensional weights established on trial $n-1$ (weighting of the target dimension) into trial $n$. The present finding that a redundant target preceding a singly, color- or orientation-defined target (RC or RO trial sequence) causes some RT cost (which may be less than a preceding target defined in a different dimension; OC sequence) can also be explained within this framework by assuming that the nonrepeated dimension of the redundant target (e.g., orientation) attracts some of 
the weight away from the repeated dimension (e.g., color). Thus, the repeated dimension would start trial $n$ at a disadvantage relative to a same-dimension repetition (a saliency signal in the repeated dimension would receive relatively less amplification), but at an advantage relative to a dimensional change.

The cost in responding to a singly defined target when the preceding target was redundantly defined (RC and RO sequences) was almost as large as the cost when the preceding target was defined in a different dimension (OC and $\mathrm{CO}$ sequences). This suggests that the nonrepeated dimension of a redundant target on trial $n-1$ (e.g., $\mathrm{O}$ in case of an $\mathrm{RC}$ sequence or $\mathrm{C}$ in case of an $\mathrm{RO}$ sequence) exerts a lasting aftereffect, perhaps by attracting dimensional weight. Consistent with this, the second of two consecutive redundant targets (RR) was responded to faster than the second of two consecutive color (CC) or two consecutive orientation $(\mathrm{OO})$ targets, suggesting that the first of two consecutive redundant targets might optimize the dimensional weight setting for coactive processing of dimensions to occur.

RT distribution analysis (target-present trials). The redundant-target RT distributions were tested for violations of the race model inequality, to examine whether the color and orientation dimensions were processed in a parallel-coactive manner. Prior to conducting the tests, "twins" of false alarm RTs were removed from the correctresponse RT distributions, ${ }^{6}$ effectively correcting for fast correct guesses (Eriksen, 1988; Grice, Nullmeyer, \& Spiker, 1977). The tests were then carried out by calculating $P(\mathrm{RT}<t / \mathrm{C})+\mathrm{P}(\mathrm{RT}<t / \mathrm{O})$ for the set of response times, $t$, corresponding to the quantiles (i.e., the 5 th, 10 th, 15 th, etc. percentiles) of the redundant-target RT distribution ( $\mathrm{C}$ and $\mathrm{O}$ denote color and orientation targets, respectively). The race model inequality is violated if $P(\mathrm{RT}<t / \mathrm{C} \& \mathrm{O})>P(\mathrm{RT}<t / \mathrm{C})+P(\mathrm{RT}<t / \mathrm{O})$. The results of the tests are presented in Table 4 . Significant violations of the race model inequality (see Student's $T$ values with positive signs and associated $P$ values $<.05$ in Table 4) were manifest only for the constant-dimension conditions, with a somewhat more robust effect for the redcolor condition (significant or marginally significant violations for the first and fourth quantiles; see Table 4A) than for the turquoise-colorcondition (significant or marginally significant violations for the second and third quantiles; see Table 4B). Combined across the red and the turquoise constant-dimension conditions, the race model was violated significantly at the second and third quantiles $\left(T_{9}=\right.$ 2.45 and 2.60 , respectively, $p<.025)$ and marginally significantly at the third quantile $\left(T_{9}=1.45, p<.10\right)$. In contrast, in the variable-dimension condition, there was no evidence for violations of the race model inequality. ${ }^{?}$

In summary, there was evidence for parallel-coactive processing of dimensions only under constant-dimension, but not under variable-dimension, conditions. The absence of significant violations of the race model inequality under variable-dimension conditions may be due to marked imbalances in the weight setting for the two dimensions, depending on the targets on the preceding trials (see the
Intertrial Effects section, above). Furthermore, under constant-dimension conditions, there was evidence for parallel-coactive processing not only when color saliency was derived faster than orientation saliency (red-color condition), but also when orientation saliency was derived faster than color saliency (turquoise-color condition).

RT distribution analysis (constant-dimension targetabsent trials). The finding that there were significant violations of the race model inequality only under constantdimension conditions indicates caution in interpreting the expedition of redundant-target RTs: It might arise (artifactually) from the lowering of positive-response decision criteria during blocks of redundant targets, rather than from parallel-coactive processing of dimensions. However, as was discussed above (see the introduction), criterion adjustments to the stronger saliency signals in blocks of redundant targets should occur for negative as well as for positive decisions. Thus, if the violations of the race model inequality were (solely) due to lowered decision criteria, target-absent RTs, too, should violate the race-model inequality, even though there are no saliency signals that could coactivate a response. If they do not, this would provide stronger grounds for interpreting the significant violations on target-present trials in terms of coactivation of a response-relevant representation by color and orientation signals simultaneously.

As can be seen from Table 5, negative RTs showed no significant violations of the race model inequality at any quantile-in fact, rather than $P(\mathrm{RT}<t / \mathrm{C} \& \mathrm{O})$ being greater than the sum of $P(\mathrm{RT}<t / \mathrm{C})$ and $P(\mathrm{RT}<t / \mathrm{O})$, it was significantly smaller from the first quantile on ward. In other words, for negative RTs, the distribution analysis reveals a trend away from violations of the redundanttarget inequality. This dissociation between positive and negative trials reinforces the arguments in favor of parallelcoactive processing of color and orientation signals.

In summary, assuming that the response criteria were not systematically lowered during blocks of redundant targets, ${ }^{8}$ the constant-dimension results provide evidence for parallel-coactive processing of dimensions. That is, within a GS framework, the feature contrast signals generated in separate dimensions may combine to coactively boost the overall saliency signal determining target detection. One interesting question was why there was evidence of such a synergy effect only under constantdimension, but not variable-dimension, conditions. Of course, the absence of significant violations of the race model inequality under the latter conditions is only a difference in significance relative to the former; it is not a significant difference. In other words, it does not mean that there are no, or cannot be, violations of the race model inequality under variable-dimension conditions. However, the power to demonstrate violations under variabledimension conditions is reduced, relative to constantdimension conditions, for the following reason. According to the dimensional-weighting account, in variabledimension conditions, the dimensional weights are constantly "meandering" between the color and the orientation dimensions, depending on the target on the preceding 
Table 4

Test for Violation of Race Model Inequality, $P(\mathrm{RT}<t / \mathrm{C \& O})>P(\mathrm{RT}<t / \mathrm{C})+P(\mathrm{RT}<t / \mathrm{O})$, Separately for the Red- and the Turquoise-Color and the Constant- and the Variable-Dimension Conditions of Experiment 1

\begin{tabular}{|c|c|c|c|c|c|c|c|c|c|c|c|c|c|}
\hline$\underline{p_{\mathrm{r}}}$ & $t_{\mathrm{r}}$ & $p_{\mathrm{c}}$ & $p_{0}$ & $p_{\mathrm{c}}+p_{\mathrm{o}}$ & $T_{9}$ & $P$ & $\underline{p_{\mathrm{r}}}$ & $t_{\mathrm{r}}$ & $p_{\mathrm{c}}$ & $p_{\mathrm{o}}$ & $p_{\mathrm{c}}+p_{\mathrm{o}}$ & $T_{9}$ & $P$ \\
\hline \multicolumn{7}{|c|}{ A. Red Color, Constant Dimension } & \multicolumn{7}{|c|}{ C. Red Color, Variable Dimension } \\
\hline 5 & 300.50 & 1.61 & 1.26 & 2.87 & 2.28 & .024 & 5 & 318.30 & 4.32 & 1.37 & 5.69 & -0.640 & .269 \\
\hline 10 & 310.95 & 4.01 & 2.16 & 6.17 & 1.70 & .062 & 10 & 327.20 & 6.65 & 2.34 & 8.99 & 0.920 & .191 \\
\hline 15 & 318.65 & 6.68 & 3.47 & 10.15 & 1.97 & .040 & 15 & 335.20 & 10.47 & 3.84 & 14.31 & 0.370 & .360 \\
\hline 20 & 324.90 & 9.93 & 5.41 & 15.34 & 1.58 & .075 & 20 & 341.95 & 13.01 & 5.75 & 18.76 & 0.578 & .289 \\
\hline 25 & 332.45 & 12.31 & 7.31 & 19.62 & 1.20 & .132 & 25 & 346.65 & 15.06 & 7.02 & 22.08 & 1.185 & .133 \\
\hline 30 & 338.60 & 16.47 & 10.16 & 26.63 & 0.64 & .269 & 30 & 350.80 & 17.79 & 7.95 & 25.74 & 1.541 & .079 \\
\hline 35 & 343.95 & 20.47 & 11.35 & 31.82 & 0.63 & .273 & 35 & 356.25 & 21.35 & 9.96 & 31.31 & 1.032 & .165 \\
\hline 40 & 349.60 & 25.11 & 13.24 & 38.35 & 0.32 & .378 & 40 & 361.70 & 25.98 & 12.91 & 38.89 & 0.294 & .388 \\
\hline 45 & 355.00 & 29.73 & 15.52 & 45.25 & -0.05 & .480 & 45 & 366.70 & 29.93 & 14.61 & 44.54 & 0.115 & .456 \\
\hline 50 & 359.70 & 34.13 & 18.60 & 52.73 & -0.54 & .300 & 50 & 372.20 & 34.54 & 18.09 & 52.63 & -0.651 & .266 \\
\hline 55 & 364.00 & 38.19 & 20.73 & 58.92 & -0.87 & .204 & 55 & 377.80 & 39.11 & 21.85 & 60.96 & -1.316 & .111 \\
\hline 60 & 370.20 & 44.24 & 26.95 & 71.19 & -2.74 & .012 & 60 & 383.60 & 43.89 & 25.25 & 69.14 & -1.742 & .058 \\
\hline 65 & 375.95 & 48.68 & 31.39 & 80.07 & -3.70 & .003 & 65 & 388.95 & 49.33 & 28.37 & 77.70 & -2.557 & .016 \\
\hline 70 & 383.35 & 54.11 & 37.19 & 91.30 & -4.65 & .001 & 70 & 395.55 & 53.82 & 32.28 & 86.10 & -3.200 & .006 \\
\hline 75 & 390.70 & 59.67 & 43.90 & 103.57 & -5.60 & .000 & 75 & 402.15 & 58.95 & 37.19 & 96.14 & -3.754 & .003 \\
\hline 80 & 400.65 & 64.75 & 52.65 & 117.40 & -6.74 & .000 & 80 & 413.20 & 65.12 & 45.27 & 110.39 & -4.022 & .002 \\
\hline 85 & 414.85 & 72.16 & 62.67 & 134.83 & -8.85 & .000 & 85 & 424.95 & 71.84 & 53.78 & 125.62 & -5.509 & .000 \\
\hline 90 & 434.10 & 79.72 & 72.19 & 151.91 & -9.68 & .000 & 90 & 442.60 & 79.75 & 64.41 & 144.16 & -8.491 & .000 \\
\hline 95 & 463.55 & 87.84 & 82.79 & 170.63 & -11.61 & .000 & 95 & 473.35 & 88.77 & 78.18 & 166.95 & -12.93 & .000 \\
\hline \multicolumn{7}{|c|}{ B. Turquoise Color, Constant Dimension } & \multicolumn{7}{|c|}{ D. Turquoise Color, Variable Dimension } \\
\hline 5 & 318.15 & 2.09 & 2.68 & 4.77 & 0.13 & .449 & 5 & 339.15 & 1.79 & 3.11 & 4.90 & 0.101 & .461 \\
\hline 10 & 328.45 & 3.05 & 4.20 & 7.25 & 1.46 & .090 & 10 & 347.75 & 3.77 & 5.63 & 9.40 & 0.392 & .352 \\
\hline 15 & 334.65 & 3.91 & 6.40 & 10.31 & 1.88 & .047 & 15 & 356.70 & 6.43 & 8.74 & 15.17 & -0.077 & .470 \\
\hline 20 & 342.60 & 5.97 & 11.14 & 17.11 & 0.76 & .233 & 20 & 362.55 & 8.72 & 11.67 & 20.39 & -0.146 & .444 \\
\hline 25 & 349.05 & 7.60 & 14.26 & 21.86 & 0.59 & .284 & 25 & 367.70 & 10.42 & 14.34 & 24.76 & 0.109 & .458 \\
\hline 30 & 355.10 & 10.00 & 18.68 & 28.68 & 0.26 & .402 & 30 & 373.40 & 12.92 & 17.52 & 30.44 & -0.207 & .421 \\
\hline 35 & 358.70 & 11.65 & 21.91 & 33.56 & 0.27 & .397 & 35 & 377.75 & 14.30 & 20.00 & 34.30 & 0.325 & .377 \\
\hline 40 & 364.05 & 15.05 & 24.81 & 39.86 & 0.03 & .491 & 40 & 382.40 & 16.90 & 23.24 & 40.14 & -0.061 & .476 \\
\hline 45 & 368.90 & 17.74 & 29.74 & 47.48 & -0.40 & .350 & 45 & 387.25 & 19.81 & 28.31 & 48.12 & -1.141 & .142 \\
\hline 50 & 373.30 & 21.80 & 33.28 & 55.08 & -0.80 & .223 & 50 & 392.65 & 23.48 & 34.14 & 57.62 & -1.706 & .061 \\
\hline 55 & 378.30 & 26.89 & 37.39 & 64.28 & -1.39 & .100 & 55 & 397.25 & 27.87 & 38.21 & 66.08 & -2.596 & .015 \\
\hline 60 & 384.65 & 33.47 & 41.30 & 74.77 & -2.01 & .038 & 60 & 402.80 & 31.89 & 41.91 & 73.80 & -3.077 & .007 \\
\hline 65 & 391.35 & 39.29 & 47.25 & 86.54 & -3.04 & .007 & 65 & 407.95 & 37.39 & 45.94 & 83.33 & -3.812 & .002 \\
\hline 70 & 398.90 & 45.18 & 52.43 & 97.61 & -3.47 & .004 & 70 & 413.95 & 41.40 & 50.58 & 91.98 & -4.064 & .002 \\
\hline 75 & 408.30 & 53.02 & 58.70 & 111.72 & -4.34 & .001 & 75 & 422.15 & 46.94 & 56.79 & 103.73 & -5.007 & .001 \\
\hline 80 & 415.85 & 58.20 & 64.40 & 122.60 & -5.75 & .000 & 80 & 431.65 & 53.71 & 64.83 & 118.54 & -7.390 & .000 \\
\hline 85 & 429.50 & 65.41 & 71.65 & 137.06 & -6.71 & .000 & 85 & 444.10 & 63.76 & 73.25 & 137.01 & -13.03 & .000 \\
\hline 90 & 463.95 & 76.47 & 82.63 & 159.10 & -8.07 & .000 & 90 & 467.45 & 74.90 & 81.70 & 156.60 & -12.72 & .000 \\
\hline 95 & 518.85 & 85.52 & 89.83 & 175.35 & -10.32 & .000 & 95 & 512.40 & 84.03 & 88.12 & 172.15 & -13.96 & .000 \\
\hline
\end{tabular}

Note-Significant violations of race model inequality are indicated by positive $T$ values and associated $P$ values $<.05$. $p_{\mathrm{r}}$, quantiles of redundanttarget RT distribution; $t_{\mathrm{r}}$, times corresponding to quantiles of redundant-target RT distribution; $p_{\mathrm{c}}, P(\mathrm{RT}<t / \mathrm{C}) ; p_{\mathrm{o}}, P(\mathrm{RT}<t / \mathrm{O}) ; p_{\mathrm{c}}+p_{\mathrm{o}}, P(\mathrm{RT}<$ $t / \mathrm{C})+P(\mathrm{RT}<t / \mathrm{O}) ; T_{9}$, Student's $t(d f=9) ; P$, one-tailed probability associated with Student's $t$.

trial. Thus, for example, a color target on the preceding trial will attract weight away from the orientation dimension and toward the color dimension. Consequently, the feature contrast signal generated by a color target on the current trial would be amplified, whereas that produced by an orientation target would be attenuated at the level of the overall activation map-consistent with the intertrial effects reported above. This would also apply to a redundant target on a given trial, which generates both a color and an orientation feature contrast signal. However, when there are large imbalances between the strengths of the two signals, any synergy effects between them would be reduced (similar to when one signal is greatly delayed relative to the other). Thus, in variabledimension conditions, violations of the race model inequality would be harder to demonstrate, owing to constantly changing imbalances in the weights assigned to the color and the orientation dimensions, modulating the strengths of their feature contrast signals on the overall activation map. However, under constant-dimension conditions, the weights would be better balanced between the relevant dimensions, providing more favorable conditions for their signals to coactivate units at the level of the overall activation (saliency) map.

A further finding was that there was evidence for parallel-coactive processing regardless of whether orientation saliency was derived faster than color saliency or vice versa. This suggests that the coactivation stage in the present experiments is separate from the visual binding stage studied by Moutoussis and Zeki (1997). They reported that, at the binding stage, color information is perceptually combined with noncolor (i.e., in their experiments, motion direction) information leading color by some $70-80 \mathrm{msec}$. If the same interactive stage had been 
Table 5

Test for Violation of Race Model Inequality, $P(\mathrm{RT}<t / \mathrm{C \& O})>P(\mathrm{RT}<t / \mathrm{C})+P(\mathrm{RT}<t / \mathrm{O})$, for the Negative Responses Combined Across the Red-and the Turquoise-Color Constant-Dimension Conditions of Experiment 1

\begin{tabular}{rcccccc}
\hline$p_{\mathrm{r}}$ & $t_{\mathrm{r}}$ & $p_{\mathrm{c}}$ & \multicolumn{1}{c}{$p_{\mathrm{o}}$} & $p_{\mathrm{c}}+p_{\mathrm{o}}$ & \multicolumn{1}{c}{$T_{9}$} & $P$ \\
\hline 5 & 342.35 & 5.48 & 4.16 & 9.64 & -3.533 & .003 \\
10 & 351.85 & 8.43 & 6.69 & 15.11 & -3.130 & .006 \\
15 & 359.15 & 12.60 & 10.31 & 22.91 & -4.571 & .001 \\
20 & 365.65 & 15.92 & 14.51 & 30.42 & -4.540 & .001 \\
25 & 371.92 & 19.54 & 17.95 & 37.49 & -5.305 & .000 \\
30 & 377.70 & 23.28 & 20.59 & 43.87 & -5.030 & .001 \\
35 & 382.90 & 27.20 & 24.22 & 51.41 & -5.706 & .000 \\
40 & 388.95 & 32.31 & 29.46 & 61.77 & -7.079 & .000 \\
45 & 394.80 & 36.03 & 33.35 & 69.38 & -7.910 & .000 \\
50 & 402.10 & 43.32 & 39.30 & 82.62 & -8.365 & .000 \\
55 & 408.08 & 47.18 & 43.95 & 91.13 & -10.667 & .000 \\
60 & 414.18 & 51.39 & 47.98 & 99.37 & -11.405 & .000 \\
65 & 422.40 & 56.45 & 54.28 & 110.72 & -13.868 & .000 \\
70 & 430.92 & 62.00 & 59.73 & 121.73 & -12.501 & .000 \\
75 & 441.60 & 67.54 & 65.49 & 133.03 & -13.850 & .000 \\
80 & 454.95 & 74.39 & 72.02 & 146.41 & -18.697 & .000 \\
85 & 470.05 & 79.04 & 77.96 & 156.99 & -23.087 & .000 \\
90 & 508.00 & 88.09 & 86.73 & 174.82 & -42.181 & .000 \\
95 & 559.92 & 93.60 & 93.26 & 186.86 & -95.580 & .000 \\
\hline
\end{tabular}

Note-Significant violations of the race model inequality would be indicated by positive $T$ values and associated $P$ values $<.05 . p_{\mathrm{r}}$, quantiles of redundant-target RT distribution; $t_{\mathrm{r}}$, times corresponding to quantiles of redundant-target RT distribution; $p_{\mathrm{c}}, P(\mathrm{RT}<t / \mathrm{C}) ; p_{\mathrm{o}}, P(\mathrm{RT}<$ $t / \mathrm{O}) ; p_{\mathrm{c}}+p_{\mathrm{o}}, P(\mathrm{RT}<t / \mathrm{C})+P(\mathrm{RT}<t / \mathrm{O}) ; T_{9}$, Student's $t(d f=9) ; P$, one-tailed probability associated with Student's $t$.

implicated in the present experiments, the violation of the race model inequality should have been more marked in the turquoise-color condition (with orientation leading color) than in the red-color condition (in anything, the reverse is true). Assuming that orientation behaves like motion direction (used by Moutoussis \& Zeki, 1997) at the perceptual-binding stage, the present results would imply that signals from different dimensions may interact at a prebinding stage, probably a stage at which overall stimulus saliencies are computed.

Error analysis. Table 6 presents the error rates in the variable-dimension condition of Experiment 1. Although the error rates were low overall $(1.15 \%)$, the miss rates were significantly greater when targets were defined singly by either color or orientation rather than redundantly by both color and orientation ( $1.10 \%$ vs. $0.35 \%)$. [An ANOVA of the target misses, with the main terms for color condition (red or turquoise) and target definition (nonredundant or redundant), revealed the effect of target definition to be significant; $F(2.0,18.0)=11.28$, $M S_{\mathrm{e}}=0.348, p<.001$; Huynh-Feldt corrected degrees of freedom.] The redundant-target advantage under conditions of dimensional variability means that targets defined in two dimensions are both detected more rapidly and less likely to be missed than targets defined in one dimension only. Although only $1 \%-2 \%$ of singly defined targets were missed, redundantly defined targets were virtually never missed (which could prove significant in applied contexts with high costs associated with target misses). In the constant-dimension condition, the miss and false alarm rates averaged $0.62 \%$ and $0.48 \%$, respectively, and exhibited no significant differences between the nonredundant and the redundant target definition conditions (misses, $0.69 \%$ vs. $0.49 \%$; false alarms, $0.50 \%$ vs. $0.46 \%$ ) and the red- and the turquoise-color conditions.

Summary of results and discussion. (1) Experiment 1 produced mean RT gains for redundantly defined targets relative to both orientation- and color-defined targets. (2) Mean RT redundancy gains were evident both when the target-defining dimension was constant and when it was variable. The mean RT redundancy gains in the constant-dimension condition argue against target detection's being based on the serial processing of potentially target-defining dimensions. (3) When the targetdefining dimension was variable, the RT to a given target was dependent on the target on the preceding trial, so that same-dimension targets were responded to faster than different-dimension targets, consistent with the target on a given trial biasing the selection weight assigned to a given dimension. Furthermore, there was evidence suggesting that the nonrepeated dimension of a redundant target on trial $n-1$ exerts a lasting aftereffect, perhaps by attracting dimensional weight, and that, in addition, the first of two repeated redundant targets optimizes the dimensional weight setting for dimensionally coactive processing of the second redundant target. (4) Analyses of the RT distributions provided evidence, under constantdimension conditions, for redundantly defined targets' violating the race model inequality - that is, for both components of redundant targets' influencing a common target detection mechanism in a parallel and coactive fashion. (No violations of the race model inequality were evident when no target was present, even though targetabsent responses exhibited a mean RT "redundancy" gain.) However, no violations of the race model inequality were found when the target-defining dimension was variable, likely owing to constantly changing imbalances in the attention weights associated with the alternative dimensions. (5) The error rates were very low overall. Nevertheless, under variable-dimension conditions, redundantly defined targets were hardly ever missed.

\section{EXPERIMENT 2}

One of the most interesting findings of Experiment 1 was that the race model inequality was violated significantly only under constant-dimension conditions, whereas there were no significant violations under variable-

Table 6

Proportions of False Alarms and Misses (in Percentages) in the Variable-Dimension Condition of Experiment 1

\begin{tabular}{|c|c|c|c|c|c|c|c|c|}
\hline & \multicolumn{8}{|c|}{ Condition } \\
\hline & \multicolumn{4}{|c|}{ Red } & \multicolumn{4}{|c|}{ Turquoise } \\
\hline & C & $\mathrm{O}$ & $\mathrm{R}$ & Mean & C & $\mathrm{O}$ & $\mathrm{R}$ & Mean \\
\hline $\begin{array}{l}\text { False alarms } \\
\text { Misses }\end{array}$ & 0.89 & 1.31 & 0.30 & $\begin{array}{l}1.42 \\
0.83\end{array}$ & 1.44 & 0.76 & 0.39 & $\begin{array}{l}1.48 \\
0.86\end{array}$ \\
\hline
\end{tabular}

Note $-\mathrm{C}, \mathrm{O}$, and R: color, orientation, and redundant-target definition. 
dimension conditions. The latter finding appears to be at variance with Mordkoff and Yantis (1993), who have reported evidence for parallel-coactive processing of color and form, using a procedure in which redundant and nonredundant targets were presented in randomized order. The present results would also appear to undermine one of the central conclusions of Mordkoff et al. (1990). Their observers were presented with displays of up to six items and had to discern the presence of a color-form conjunction target. On redundant-target trials, two targets were presented at separate display locations. Mordkoff et al. (1990) found violations of the race model inequality, arguing against visual search models such as GS that assume a strictly serial stage (with search limited to one item at a time). Mordkoff et al. (1990) assumed that their findings would generalize to "typical" search displays with large numbers of items ( $>6$ items). The present results could therefore be taken to question this assumption. However, before examining whether display size is the critical variable distinguishing between the present findings and those of Mordkoff and his colleagues, other possibilities ought to be explored.

One possibility, suggested by the intertrial transition effects in Experiment 1, is that intertrial weight shifts obscured the manifestation of violations of the race model inequality under variable-dimension conditions. According to the dimension-weighting account, random changes in the target definition across trials (from one single dimension to another or from a single dimension to both dimensions, and vice versa) are likely to alter the weight setting in such a way that a handicap is provided for one of the single dimensions in activating the detection response conjointly with the other dimension. For example, a color target on the preceding trial $(n-1)$ will attract weight away from the orientation dimension and toward the color dimension. Consequently, the feature contrast signal generated by a color target on the current trial $(n)$ is amplified, whereas that produced by an orientation target is attenuated at the level of the overall saliency map. This also applies to the color and orientation contrast signals generated by a redundant target on trial $n$. When there are large imbalances between the strengths of the two signals, any coactivation effects would be reduced, because detection would be mediated mainly, or entirely, by the dominant signal. Consequently, under variabledimension conditions, violations of the race model inequality would be hard to demonstrate. In contrast, under constant-dimension conditions, the weights on redundanttarget trials would be more balanced between the color and the orientation dimensions, permitting their contrast signals to interact.

This account predicts that, under variable-dimension conditions, significant violations of the race model inequality should be evident when the tests are based on identical-repetition trials that most closely match the consistent-dimension condition-specifically, analysis of the RT distributions for the second of two redundant targets $(\mathrm{R} R)$, as compared with the second of two color $(\mathrm{C} C)$ and the second of two orientation $(\mathrm{O} O)$ targets: $P(\mathrm{RT}<t / \mathrm{R} R) \leq P(\mathrm{RT}<t / \mathrm{C} C)+P(\mathrm{RT}<t / \mathrm{O} O)$. In contrast, there should be no violations for the first of two redundant targets $(R \mathrm{R})$, as compared with the first of two color $(C C)$ and the first of two orientation $(O O)$ targets (where the first target is hardly ever a repetition of the immediately preceding target, matching the intertrial contingencies under variable-dimension conditions). Unfortunately, there were insufficient numbers of identicalrepetition trials in the variable-dimension condition of Experiment 1, so that the appropriate tests for violations of the race model inequality could not be performed. Thus, to test the above predictions, Experiment 2 repeated the variable-dimension condition of Experiment 1, but with the number of trials doubled to ensure that there was a sufficiently large number of identical target repetitions on consecutive trials for violations of the race model inequality to be examined.

The possibility of intertrial effects' obscuring coactive RT redundancy gains under variable-dimension conditions bears on the question of whether response criterion adjustments were responsible for the violations of the race model inequality in the constant-dimension condition of Experiment 1 . As was discussed above, if response criterion adjustments had indeed been responsible for producing these violations, without color and orientation saliency signals combining to coactivate detection responses, violations should also have been manifest on negative (target-absent) trials, on which there could not be any coactivation, by definition. But there were no violations on negative trials. However, the absence of significant violations on negative trials may not be deemed conclusive in ruling out confounding response criterion effects under constantdimension conditions. Therefore, the analyses of RTs to first and second targets on identical-repetition trials that were planned in Experiment 2 were also intended to shed further light on the possibility of artifactual violations of the race model inequality by response criterion adjustments. Since repeated redundant targets are rare under the variable-dimension conditions employed in Experiment 2, strategic response criterion adjustments would be unlikely to account for significant violations of the race model inequality, because observers would have no grounds to expect that a redundant target would be followed immediately by another redundant target.

\section{Method}

Participants. Twelve observers participated in Experiment 2. Their ages ranged between 20 and 34 years (average, 24.1); 7 were female, and 5 male. All the observers had normal vision (including color vision).

Stimuli. The stimuli were the same as those in the red-color condition of Experiment 1. The target was marked by a red square frame (color target), a green diamond frame, or a red diamond frame (redundant condition). 
Design and Procedure. The target-def ining dimension was variable during trial blocks. To ensure that the frequencies of colorcolor [CC], orientation-orientation [OO], and redundant-redundant (RR) target repetitions were high enough to permit tests of the race model inequality to be performed, the number of trials was doubled, in comparison with Experiment $1(2 \times 600$ trials $)$. Otherwise, trial sequences for particular participants were generated according to the same procedure as that in Experiment 1. This procedure simply specified that the proportion of negative (target-absent) trials should be $50 \%$ and the proportion of positive trials should be $16.67 \%$ for each of the color-, orientation-, and redundant-target conditions. The trials thus generated were then simply presented in randomized order. In Experiment 2, random orders were generated in the same way but were introduced into the experiment only if the probabilities of $\mathrm{CC}, \mathrm{OO}$, and $\mathrm{RR}$ target repetitions were sufficiently high (average probabilities of $\mathrm{CC}, \mathrm{OO}$, and RR repetitions in Experiments 1 and $2,2.84 \%, 2.89 \%$, and $2.49 \%$ vs. $2.49 \%, 2.60 \%$, and $2.91 \%$; an ANOVA comparing the probabilities of identical target repetitions between the two experiments revealed no significant effects). The participants performed the 1,200 trials in two sessions, each consisting of nine blocks of 60 trials (plus an initial practice block).

\section{Results and Discussion}

Mean RT redundancy gain. Table 7 presents the mean correct RTs for the color-, orientation-, and redundanttarget conditions, separately for the first and the second targets of identical-repetition doublets (CC, OO, RR). A repeated measures ANOVA, with main terms for target definition (color, orientation, or redundant) and doublet position (first or second), revealed both main effects to be significant [target definition, $F(2,22)=29.906, M S_{\mathrm{e}}=$ $414.59, p<.001$; doublet position, $F(1,11)=18.327$; $\left.M S_{\mathrm{e}}=288.50, p<.001\right]$. Redundant targets were responded to faster than singly defined targets, even when compared with the, on average, faster of the two singledimension targets (color) $\left[F(1,11)=11.15, M S_{\mathrm{e}}=550.8\right.$, $p<.01$; i.e., the Biederman-Checkosky gain was significant]. Furthermore, targets in the second doublet position were responded to faster than those in the first position. Although the mean RT redundancy gain was numerically larger for second- than for first-position targets (Biederman-Checkosky gains, 26 vs. $19 \mathrm{msec}$ ), as was expected, the two-way interaction was not significant $(F<1)$. The mean RT redundancy gains estimated according to the Miller-Lopes procedure were also reliable

Table 7

Mean Correct Reaction Times (RTs, in Milliseconds) and Associated Miss Rates (MRs, in Percentages) to Color-Defined, Orientation-Defined, and Redundantly Defined Targets, Separately for the First and the Second of Doublets of Repeated Targets in Experiment 2

\begin{tabular}{|c|c|c|c|c|c|c|c|c|}
\hline \multirow[b]{2}{*}{ Target } & \multicolumn{2}{|c|}{$\mathrm{CC}$} & \multicolumn{2}{|c|}{$\mathrm{OO}$} & \multicolumn{2}{|c|}{ RR } & \multirow[b]{2}{*}{ Gain B\&C } & \multirow[b]{2}{*}{ Gain M\&L } \\
\hline & RT & MR & RT & $\overline{M R}$ & RT & $\overline{M R}$ & & \\
\hline First & 405.25 & 1.0 & 431.00 & 2.5 & 386.17 & 0.8 & 19.08 & 21.54 \\
\hline Second & 392.42 & 1.0 & 412.33 & 2.1 & 366.25 & 0.5 & 26.17 & 26.88 \\
\hline
\end{tabular}

Note-For comparison, the mean correct target-absent RT was $419.17 \mathrm{msec}$, and the mean false alarm rate was $1.36 \%$.CC, color-color; $\mathrm{OO}$, orientation-orientation; RR, redundant-redundant; Gain ${ }^{\mathrm{B} \& \mathrm{C}}$, RT redundancy gain relative to the on average fastest single-dimension target condition (Biederman \& Checkosky, 1970); Gain ${ }^{\text {M\&L }}$, RT redundancy gain computed according to Miller and Lopes (1988).
Table 8

Mean Correct Reaction Times (in Milliseconds) to Color-Defined and Orientation-Defined Targets on Trial $n$, Depending on Whether the Target on Trial $n-1$ was Defined in the Same Dimension, in Both Dimensions (Redundant), or in a Different Dimension, in Experiment 2

\begin{tabular}{lccc}
\hline & \multicolumn{3}{c}{ Target $n-1$} \\
\cline { 2 - 4 } Target $n$ & Same & Redundant & Different \\
\hline Color & 391.7 & 395.4 & 409.7 \\
Orientation & 412.8 & 437.5 & 444.3 \\
\hline
\end{tabular}

overall $\left[F(1,11)=11.901, M S_{\mathrm{e}}=590.9, p<.005\right]$ but exhibited no significant difference between second- and firstposition targets (Miller-Lopes gains, 27 vs. $22 \mathrm{msec}$ ).

Intertrial effects. Table 8 presents the mean correct RTs to color-defined and orientation-defined targets on a given trial $(n)$, depending on whether the target on the preceding trial $(n-1)$ was singly defined in the same dimension, redundantly defined in both dimensions (redundant), or singly defined in a different dimension. A repeated measures ANOVA with main terms for target $n$ (target dimension on trial $n$, color or orientation) and target $n-1$ (target dimension on trial $n-1$, same, redundant, or different) revealed the main effect of target $n-1$ to be significant $\left[F(2,22)=11.58, M S_{\mathrm{e}}=319.3, p<.001\right]$ : RTs were significantly faster when the preceding target was defined within the same dimension than when it was redundantly defined [402.3 vs. $416.5 \mathrm{msec} ; F(1,11)=$ 24.96, $\left.M S_{\mathrm{e}}=588.1, p<.001\right]$, and RTs tended to be faster when the preceding target was redundantly defined than when it was defined within a different dimension $\left[416.5\right.$ vs. $427.0 \mathrm{msec} ; F(1,11)=4.27, M S_{\mathrm{e}}=626.8, p<$ .063]. (There was also a significant main effect of target $n\left[F(1,11)=68.7, M S_{\mathrm{e}}=277.597, p<.001\right]$, with faster RTs to color-defined than to orientation-defined targets.) This pattern of intertrial effects, characterized by costs in responding to singly defined targets when the preceding target was defined in a different dimension and, less marked, when it was redundantly defined, is consistent with that observed in Experiment 1. That there were costs in responding to singly defined targets when the preceding target was redundantly defined suggests that the dimension of a redundant target (trial $n-1)$ that is not repeated (trial $n$ ) exerts a lasting aftereffect on the detection of a target (trial $n$ ) singly defined within the repeated dimension.

Furthermore, RTs to redundantly defined targets on a given trial $(n)$ were significantly faster when the preceding target $(n-1)$ was redundantly defined than when it was defined in a single dimension [366.6 vs. $384.4 \mathrm{msec}$; $t(11)=3.44, p<.01]$. This is consistent with the idea that the first redundant target optimizes the dimensional weight setting for coactive processing of dimensions to occur.

RT distribution analysis. The redundant-target RT distributions were tested for violations of the race model inequality, $P(\mathrm{RT}<t / \mathrm{C} \& \mathrm{O})>P(\mathrm{RT}<t / \mathrm{C})+P(\mathrm{RT}<t / \mathrm{O})$, to examine whether there were coactivation effects between the color and the orientation feature contrast signals. Table 9 presents the results of the tests, performed sepa- 
Table 9

Test for Violation of Race Model Inequality, $P(\mathrm{RT}<t / \mathrm{C \& O})>\mathrm{P}(\mathrm{RT}<t / \mathrm{C})+P(\mathrm{RT}<t / \mathrm{O})$, Separately for the First and the Second of Doublets of Repeated Targets in Experiment 2

\begin{tabular}{|c|c|c|c|c|c|c|}
\hline$p_{\mathrm{r}}$ & $t_{\mathrm{r}}$ & $p_{\mathrm{c}}$ & $p_{\mathrm{o}}$ & $p_{\mathrm{c}}+p_{\mathrm{o}}$ & $T_{11}$ & $P$ \\
\hline \multicolumn{7}{|c|}{ A. First Target } \\
\hline 5 & 287.17 & 2.07 & 1.11 & 3.18 & 1.639 & .065 \\
\hline 10 & 300.75 & 4.32 & 2.65 & 6.97 & 1.542 & .076 \\
\hline 15 & 317.33 & 8.16 & 3.27 & 11.42 & 1.383 & .097 \\
\hline 20 & 327.33 & 14.01 & 5.00 & 19.00 & 0.239 & .408 \\
\hline 25 & 335.25 & 18.59 & 6.39 & 24.98 & 0.004 & .499 \\
\hline 30 & 341.00 & 21.57 & 7.72 & 29.29 & 0.161 & .438 \\
\hline 35 & 347.50 & 25.50 & 11.05 & 36.55 & -0.333 & .373 \\
\hline 40 & 356.33 & 32.57 & 13.26 & 45.82 & -1.188 & .130 \\
\hline 45 & 364.42 & 36.85 & 20.95 & 57.80 & -2.019 & .035 \\
\hline 50 & 371.17 & 39.66 & 23.98 & 63.64 & -2.101 & .030 \\
\hline 55 & 376.58 & 44.3 & 27.56 & 71.90 & -2.445 & .017 \\
\hline 60 & 382.25 & 48.56 & 29.94 & 78.50 & -2.800 & .009 \\
\hline 65 & 389.08 & 54.56 & 34.37 & 88.93 & -3.528 & .003 \\
\hline 70 & 398.50 & 61.81 & 40.51 & 102.31 & -4.221 & .001 \\
\hline 75 & 409.58 & 67.58 & 46.44 & 114.02 & -4.465 & .001 \\
\hline 80 & 417.42 & 70.35 & 51.11 & 121.46 & -5.080 & .000 \\
\hline 85 & 437.25 & 77.36 & 62.92 & 140.28 & -9.105 & .000 \\
\hline 90 & 464.00 & 83.21 & 72.98 & 156.19 & -12.343 & .000 \\
\hline 95 & 506.50 & 88.51 & 85.36 & 173.88 & -18.160 & .000 \\
\hline \multicolumn{7}{|c|}{ B. Second Target } \\
\hline 5 & 282.00 & 2.05 & 0.51 & 2.55 & 2.392 & .018 \\
\hline 10 & 295.33 & 4.32 & 1.23 & 5.55 & 2.711 & .010 \\
\hline 15 & 305.83 & 7.87 & 2.36 & 10.23 & 2.407 & .018 \\
\hline 20 & 310.50 & 9.90 & 2.61 & 12.50 & 3.590 & .002 \\
\hline 25 & 318.17 & 14.19 & 4.77 & 18.96 & 2.855 & .008 \\
\hline 30 & 325.08 & 18.52 & 6.78 & 25.31 & 1.685 & .060 \\
\hline 35 & 331.00 & 22.52 & 8.57 & 31.09 & 1.322 & .107 \\
\hline 40 & 337.92 & 27.79 & 11.84 & 39.63 & 0.102 & .461 \\
\hline 45 & 346.50 & 32.74 & 16.92 & 49.66 & -1.129 & .142 \\
\hline 50 & 352.58 & & & & & .097 \\
\hline 55 & 358.83 & 41.83 & 21. & 63.78 & -1.881 & .043 \\
\hline 60 & 365.33 & 46.05 & 27.33 & 73.38 & -2.377 & .019 \\
\hline 65 & 373.58 & 52.33 & 33.77 & 86.09 & -3.694 & .002 \\
\hline 70 & 382.83 & 59.88 & 39.63 & 99.52 & -4.943 & .000 \\
\hline 75 & 391.17 & 64.06 & 45.27 & 109.33 & -5.047 & .000 \\
\hline 80 & 402.25 & 69.52 & 52.61 & 122.13 & -5.782 & .000 \\
\hline 85 & 412.50 & 73.99 & 59.57 & 133.56 & -7.025 & .000 \\
\hline 90 & 436.17 & 78.59 & 68.95 & 147.55 & -7.808 & .000 \\
\hline 95 & 463.17 & 85.37 & 79.52 & 164.89 & -12.129 & .000 \\
\hline
\end{tabular}

Note-Significant violations of the race model inequality are indicated by positive $T$ values and associated $P$ values $<.05 . p_{\mathrm{r}}$, quantiles of redundant-target RT distribution; $t_{\mathrm{r}}$, times corresponding to quantiles of redundant-target RT distribution; $p_{\mathrm{c}}, P(\mathrm{RT}<t / \mathrm{C}) ; p_{\mathrm{o}}, P(\mathrm{RT}<t / \mathrm{O})$; $p_{\mathrm{c}}+p_{\mathrm{o}}, P(\mathrm{RT}<t / \mathrm{C})+P(\mathrm{RT}<t / \mathrm{O}) ; T_{11}$, Student's $t(d f=11) ; P$, onetailed probability associated with Student's $t$

rately for the first (Table 9A) and the second (Table 9B) targets of identical-repetition doublets in Experiment 2. For the first target, there were no significant violations of the race model inequality (although there were marginally significant violations for Quantiles 1-3, see Table 9A). In contrast, significant violations of the race model inequality were manifest for the second target (significant violations for Quantiles 1-5; see Table 9B). Thus, as was expected, there was significant evidence for parallelcoactive processing of color and orientation feature contrast signals only for the second of two repeated targets, when the dimensional weight setting was optimized for redundant-target detection (see the Intertrial Effects section, above).

Error analysis. A repeated measures ANOVA of the arcsine-transformed miss rates (see Table 7), with main terms for target definition (color, orientation, or redundant) and doublet position (first or second), revealed significant main effects for doublet position $[F(1,11)=6.53$; $\left.M S_{\mathrm{e}}=0.0047, p<.05\right]$ and target definition $[F(2,22)=$ $7.13, M S_{\mathrm{e}}=0.226, p<.004$; the interaction was not significant $(F<1)]$. The miss rates were reduced overall for the second of two repeated targets, as was predicted. Furthermore, the miss rates were lowest for redundant targets [significant contrast for redundant vs. orientation targets, $\left.F(1,11)=9.99, M S_{\mathrm{e}}=0.508, p<.01\right]$, consistent with Experiment 1.

In summary, Experiment 2 showed that dimensional variability of intertrial transitions between consecutive targets obscures violations of the race model inequality, as would be predicted by the dimension-weighting account. Under conditions of dimensional intertrial variability, when RTs to the first of two redundant $(R \mathrm{R})$ targets were compared against RTs to the first of two color $(C \mathrm{C})$ and the first of two orientation $(O \mathrm{O})$ targets, the race model inequality was not significantly violated. In contrast, there were significant violations under conditions of dimensional intertrial constancy, when RTs to the second of two consecutive redundant $(\mathrm{R} R)$ targets were compared against RTs to the second of two color $(\mathrm{CC})$ and the second of two orientation $(\mathrm{O} O)$ targets. It is unlikely that these violations are due to strategic adjustments of the response criterion when the target-defining dimension remains constant across trials, because dimensional constancy was much less likely than dimensional change. Rather, dimension-specific feature contrast signals can combine to coactivate detection responses not only under constant-dimension conditions, but also under variabledimension conditions, but under the latter conditions coactivation effects are hard to demonstrate because of the high frequency of dimensional-change trials. On such trials, processing is likely to start with a setting of dimensional weights that is nonoptimal for redundant-target detection - that is, weights biased toward one of the dimensions defining a redundant target—so that the feature contrast signal generated by the other dimension is too weak at the integration stage to significantly expedite target detection.

\section{GENERAL DISCUSSION}

\section{Summary of Results}

Dimensionally redundant target coding (color and orientation dimensions) expedited target detection, while at the same time reducing target miss rates. Importantly, redundant-target coding expedited RTs across the whole range of RTs, including the fast end of the RT distribution, rather than just improving the mean RT (coactive gains significantly violating the race model inequality). Such coactive gains were most reliably achieved when 
targets were defined consistently in a dimensionally redundant manner, rather than when the definition was variable, changing unpredictably between redundantand nonredundant-target trials.

\section{Theoretical Implications}

The violations of the race model inequality demonstrated in the present experiments provide strong evidence against serial models (e.g., Grossberg et al., 1994; Treisman, 1988) and parallel-race models of cross-dimension feature search and evidence in favor of parallel-coactive processing of target dimensions. This result may be best explained in terms of search models that assume that target detection is based on a master map representation of integrated (summed) dimension-specific saliency signals (e.g., GS; Wolfe, 1994), which would permit both components of redundant targets to influence a common target detection stage in a coactive fashion.

Consistent with this, parallel-coactive processing was evident not only when color saliency was derived faster than orientation saliency, but also when orientation saliency was derived faster than color saliency (Experiment 1; although, of course, parallel-coactive signal integration would be expected to fail if the differential coding efficiency between the redundant dimensions becomes too large). The finding that the violations of the race model inequality were, if anything, more robust when color saliency was leading orientation saliency (red-color condition of Experiment 1) suggests that the signal integration stage in the present experiments is separate from the visual-binding stage studied by Moutoussis and Zeki (1997), at which color information is combined with noncolor information leading color by some $70-80 \mathrm{msec}$. The present results would imply that signals from different dimensions may interact at a prebinding stage. According to the dimension-weighting account, this stage is that at which overall stimulus saliencies are computed.

However, simple models assuming integration of dimension-specific saliency signals at a master map stage (such as earlier versions of GS) make it difficult to explain why reliable violations of the race model inequality were evident in the present experiments only when redundant-target definition was constant within blocks of trials (Experiment 1) or across consecutive trials (Experiment 2), rather than changing randomly between redundant and single-dimension definition. This dissociation can be explained when the simple model is extended by the assumption that dimension-specific saliency signals are weighted as they are transmitted to the master map of activations (Found \& Müller, 1996; Müller et al., 1995). Experiment 2 provided evidence that changes in the target-defining dimension(s) across consecutive trials give rise to shifts and imbalances in the weight setting for the redundant dimensions, affecting the strength (and/ or rise time) of their feature contrast signals at the integration stage. When there are large weight imbalances between the two signals, any coactivation effects will be reduced on redundant-target trials. Therefore, under variable-dimension conditions, violations of the race model inequality are harder to demonstrate than under constant-dimension conditions (in which the weights are better balanced between the redundant dimensions, permitting their saliency signals to interact).

Importantly, Experiment 2 demonstrated that effects of dimensional variability or constancy can also be obtained within a single experiment in which change of a target-defining dimension across trials is much more likely than no change. This effectively rules out the possibility that violations of the race model inequality under constantdimension conditions are due to adjustments of response thresholds ${ }^{9}$ and supports the alternative position that redundantly defined targets produce stronger signals and/or a faster rise in signal strengths at the detection stage. The present findings are, therefore, not at variance with those reported by Mordkoff and Yantis (1993), who had previously demonstrated parallel-coactive processing of color and form under variable-dimension conditions. Rather, the present results extend their findings by specifying the conditions under which coactive RT redundancy gains are optimally obtained, in addition to showing that their findings do extend to typical search displays with large numbers of items.

It has been proposed above that feature contrast signals from different dimensions may interact at a prebinding stage. Although this is not contentious, there has recently been some controversy concerning the locus of dimensional-uncertainty effects (e.g., Müller et al., 1995; Treisman, 1988) and, by extension, redundant-target definition effects (present study). On the basis of such theories as GS (Koch \& Ullman, 1985; Wolfe, 1994), the dimension-weighting account of Müller and his colleagues assumes that dimensional-uncertainty effects and redundant-target coding effects reflect the operation of a supradimensional saliency representation, the overall activation map, which serves two functions: to support target detection and to guide focal attention to the target. Concerning the latter, Found and Müller (1996) suggested that detection responses may be based "more or less directly on ... the "master map of activations/ locations' (coding the location of objects to be attended and responded to) without waiting for complete object knowledge to become available" (p. 99). On this account, dimensional-uncertainty and redundant-target coding effects are essentially perceptual in nature. In contrast, Cohen and Magen (1999) have proposed a response-based account that builds on the cross-dimensional response selection model of Cohen and Shoup (1997). According to this account, the various dimensional processing modules (e.g., color or orientation) possess separate response selection mechanisms. Effects of dimensional uncertainty can then be explained as follows: "An intradimensional [search] task [target-defining dimension fixed] requires the use of a single response selection mechanism. By contrast, cross-dimensional tasks [target-defining dimension variable] require the use of multiple response selection mechanisms" (Cohen \& Magen, p. 292). A similar account could be derived for the redundant-target coding effects demonstrated in the present experiments 
(and the experiments of Mordkoff \& Yantis, 1993): Redundantly defined targets activate separate dimensionspecific response selection mechanisms, which, in turn, drive a common response output stage in a parallelcoactive manner. (The account of Cohen and Magen is akin to the model of redundancy gains presented by Mordkoff and Yantis, 1993, which assumes separate decision devices within each, color and shape, dimension).

The present experiments do not permit a decision to be made between these alternatives. One difference between the dimension-weighting and the response-based accounts is that the former, but not the latter, assumes that the weighting concerns spatial search processes: dimensionspecific saliency computations or the transmission of their outputs to integrating, "overall," saliency units. Thus, one might argue that there should be no violations of the race model inequality when there is no uncertainty as to the target location-which would be at variance with Experiment 1 of Mordkoff and Yantis (1993). In this experiment, a single stimulus appeared at a fixed (central) location, and yet there were significant violations of the race model inequality when the stimulus was a dimensionally redundant target. ${ }^{10}$ However, what the dimension-weighting account actually predicts is that the coactivation effects are spatially specific - that is, the separate dimensional saliency signals must arise from corresponding locations to coactivate a common overall saliency unit. Consequently, the dimension-weighting account is not challenged by the finding of coactivation effects in paradigms with only one possible stimulus location, because the separate activations produced by a dimensionally redundant target would originate from the same location. ${ }^{11}$

One finding that could discriminate between the dimension-weighting and the response-based accounts would be that not only target detection responses, but also responses based on further analysis of the target (after its detection) are facilitated by dimensionally redundant target definition. Such an experiment was carried out by Krummenacher et al. (in press), who used the same stimulus outline frames as those employed in the present experiments, but with one or two green dials displayed in the inner circle. Observers had first to detect the target outline frame and then read off the dial positions inside and translate them into a response. This extended task was meant to take account of the fact that, in almost all work contexts, it is insufficient to simply spot an important indicator (e.g., an alarm signal); rather, the detection must be followed by a fast and accurate response to the information conveyed by the indicator. Krummenacher et al. found that, with well-practiced observers, target outline definition on two dimensions produced significant mean RT redundancy gains (whether calculated according to the Biederman \& Checkosky, 1970, procedure or the Miller \& Lopes, 1988, procedure). These gains are hard to explain in terms of dimension-specific response selection mechanisms, because the dimensionbased information necessary to detect and locate the target was irrelevant for the dial task response required. It is more plausible to attribute the expedition of the dial task RTs to enhanced target coding at the detection stage, permitting faster access to the target dial information required for response. This does not rule out that, when simple-detection decisions are to be made, reactions are driven by dimension-based response selection mechanisms, as was suggested by Cohen and Magen (1999). Further work is necessary to clarify this issue.

\section{Implications for Visual-Display Design}

The finding that dimensionally redundant target definition expedites target detection in a parallel-coactive manner, while at the same time reducing target miss rates, has direct implications for visual-display design: Redundant-target definition should be implemented whenever response speed is critical and when the cost associated with target misses is high. However, the present findings also indicate that, in order to achieve optimal, coactive, RT gains from redundant target definition, the definition scheme ought to be consistent (rather than variable), with consistency of the scheme appearing to be more important than the precise matching of how efficiently singly defined targets are detected in their individual defining dimensions.

The RT gains of adding a redundant dimension to the definition of target signals may appear to be too insubstantial to justify its implementation in most real-world tasks. However, even small RT gains can provide significant practical benefits when the high frequency with which visual search tasks are carried out is taken into account. The same arguments apply to the beneficial effect of redundant-target coding in improving response accuracy (reduced miss rates). In any case, the implementation of redundant-target coding is to be recommended for all tasks for which the rapid and accurate detection of target signals is vital.

\section{REFERENCES}

BACKS, R. W., \& WALRATH, L. C. (1995). Ocular measures of redundancy gain during visual search of color symbolic displays. Ergonomics, 38, 1831-1840.

Bacon, W. F., \& Egeth, H. E. (1994). Overriding stimulus-driven attentional capture. Perception \& Psychophysics, 55, 485-496.

Biederman, I., \& Checkosky, S. F. (1970). Processing redundant information. Journal of Experimental Psychology, 83, 486-490.

Bundesen, C. (1990). A theory of visual attention. Psychological Review, 97, 523-547.

Bundesen, C. (1998). A computational theory of visual attention. Philosophical Transactions of the Royal Society: Series B, 353, 1271-1281.

CAve, K. R., \& Wolfe, J. M. (1990). Modelling the role of parallel processing in visual search. Cognitive Psychology, 22, 225-271.

Christ, R. E. (1975). Review and analysis of color coding research for visual displays. Human Factors, 17, 542-570.

Chun, M. M., \& Wolfe, J. M. (1996). Just say no: How are visual searches terminated when there is no target present? Cognitive Psychology, 30, 39-78.

Cohen, A., \& Magen, H. (1999). Intra- and cross-dimensional visual search for single-feature targets. Perception \& Psychophysics, 61, 291-307.

Cohen, A., \& Shoup, R. (1997). Perceptual dimensional constraints on response selection processes. Cognitive Psychology, 32, 128-181.

ERIKSEN, C. W. (1988). A source of error in attempts to distinguish coactivation from separate activation in the perception of redundant targets. Perception \& Psychophysics, 44, 191-193. 
Found, A. P., \& Müller, H. J. (1996). Searching for unknown feature targets on more than one dimension: Investigating a "dimensionweighting" account. Perception \& Psychophysics, 58, 88-101.

Grice, G. R. (1968). Stimulus intensity and response evocation. Psychological Review, 75, 359-373.

Grice, G. R., Nullmeyer, R., \& Spiker, V. A. (1977). Application of variable criterion theory to choice reaction time. Perception \& Psychophysics, 22, 431-449.

Grossberg, S., Mingolla, E., \& Ross, W. D. (1994). A neural theory of attentive visual search: Interaction of boundary, surface, spatial, and object representations. Psychological Review, 101, 470-489.

Humphreys, G. W., \& Müller, H. (1993). Search via recursive rejection (SERR): A connectionist model of visual search. Cognitive Psychology, 25, 43-110.

Косн, C., \& Ullman, S. (1985). Shifts in selective visual attention: Towards the underlying neural circuitry. Human Neurobiology, 4, 219-227.

Krummenacher, J., Müller, H. J., \& Heller, D. (in press). Visual search for dimensionally redundant pop-out targets: Redundancy gains in compound tasks. Visual Congition.

Maljkovic, V., \& Nakayama, K. (1994). Priming of pop-out: I. Role of features. Memory \& Cognition, 22, 657-672.

Miller, J. (1982). Divided attention: Evidence for coactivation with redundant signals. Cognitive Psychology, 14, 247-279.

Miller, J., \& Lopes, A. (1988). Testing race models by estimating the smaller of two true mean or true median reaction times: An analysis of estimation bias. Perception \& Psychophysics, 44, 513-524.

Monk, T. H. (1976). Target uncertainty in applied visual search. Human Factors, 18, 607-612.

Mordkoff, J. T., Miller, J., \& Roch, A. C. (1996). Absence of coactivation in the motor component: Evidence from psychophysiological measures of target detection. Journal of Experimental Psychology: Human Perception \& Performance, 22, 25-41.

Mordkoff, J. T., \& Yantis, S. (1991). An interactive race model of divided attention. Journal of Experimental Psychology: Human Perception \& Performance, 17, 520-538.

MordKoff, J. T., \& YANTIS, S. (1993). Dividing attention between color and shape: Evidence of coactivation. Perception \& Psychophysics, 53, 357-366.

Mordkoff, J. T., Yantis, S., \& Egeth, H. E. (1990). Detecting conjunctions of color and form in parallel. Perception \& Psychophysics, 48, 157-168.

Moutoussis, K., \& ZeKI, S. (1997). A direct demonstration of perceptual asynchrony in vision. Proceedings of the Royal Society of London: Series B, 264, 393-399.

Müller, H. J., Heller, D., \& Ziegler, J. (1995). Visual search for singleton feature targets within and across feature dimensions. Perception \& Psychophysics, 57, 1-17.

Nothdurft, H. C. (1991). Texture segmentation and pop-out from orientation contrast. Vision Research, 33, 839-844.

Nothdurft, H. C. (1992). Feature analysis and the role of similarity in preattentive vision. Perception \& Psychophysics, 52, 355-375.

NothduRfT, H. C. (1993). The role of features in preattentive vision: Comparison of orientation, motion and color cues. Vision Research, 33, 1937-1993.

PAshler, H. (1988). Cross-dimensional interaction and texture segregation. Perception \& Psychophysics, 43, 307-318.

Segalowitz, S. J., \& GRaves, R. E. (1990). Suitability of the IBM XT, AT, and PS/2 keyboard, mouse, and game port as response devices in reaction time paradigms. Behavior Research Methods, Instruments, \& Computers, 22, 283-289.

Strayer, D. L., \& Kramer, A. F. (1994). Strategies and automaticity: II. Dynamic aspects of strategy adjustment. Journal of Experimental Psychology: Learning, Memory, \& Cognition, 20, 342-365.

TheeUwes, J. (1991). Cross-dimensional perceptual selectivity. Perception \& Psychophysics, 50, 184-193.

Theeuwes, J. (1992). Perceptual selectivity for color and form. Perception \& Psychophysics, 51, 599-606.

Treisman, A. (1988). Features and objects: The fourteenth Bartlett
Memorial Lecture. Quarterly Journal of Experimental Psychology, 40A, 201-236.

Treisman, M., \& Williams, T. C. (1984). A theory of criterion setting with an application to sequential dependencies. Psychological Review, 91, 68-111.

WiCKENS, C. D. (1992). Engineering psychology and human performance (2nd ed.). New York: Harper Collins.

Wolfe, J. M. (1994). Guided Search 2.0: A revised model of visual search. Psychonomic Bulletin \& Review, 1, 202-238.

Wolfe, J. M., Chun, M. M., \& Friedman-Hill, S. R. (1995). Making use of texton gradients: Visual search and perceptual grouping exploit the same parallel processes. In T. Papathomas \& A. Gorea (Eds.), Linking psychophysics, neuropsychology, and computational vision (pp. 189-197). Cambridge, MA: MIT Press.

Zohary, E., Shadlen, M. N., \& Newsome, W. T. (1994). Correlated neural discharge rate and its implication for psychophysical performance. Nature, 370, 140-143.

\section{NOTES}

1. Feature-specific intertrial transition effects have been reported by Maljkovic and Nakayama (1994) for the color dimension, but not for the orientation dimension. This difference was replicated by Found and Müller (1996, Experiments 2 and 3), who showed in addition that dimensional change produces greater effects than feature change, even for the color dimension. Nevertheless, the feature-specific intertrial transition effects, taken together with reports of feature-specific grouping effects for the color dimension (Nothdurft, 1992; Wolfe, Chun, \& FriedmanHill, 1995), suggest that color is a special dimension.

2. The dimension-weighting account of Müller and his colleagues is consistent with general theories of visual search, notably that developed by Bundesen (1990, 1998). See Müller et al. (1995) and Found and Müller (1996) for a discussion of their account in relation to other work on oddone-out feature search - in particular, Bacon and Egeth (1994), Maljkovic and Nakayama (1994), Nothdurft (1991, 1992), Pashler (1988), and Theeuwes $(1991,1992)$.

3. This issue is independent of the distinction between spatially parallel and serial processing of display stimuli. The issue is whether the individualdimensions on which stimuli are defined (such as their color, orientation, etc.), rather than the individual stimuli in a display, are processed simultaneously or sequentially. The former factor would affect the base RT (the $y$-intercept of the function relating search RT to display size), whereas the latter factor would affect the search rate (the slope of the search RT function).

4. Treisman (1988) explicitly assumes serial checking of dimensions: "The 'odd-one-out' [item] pops out within a single, pre-specified dimensional module, but [when the target-defining dimension is uncertain] each different module may need to be separately checked to determine which of them contains it" (p. 207). Grossberg et al. (1994), in their spatial and object search algorithm (SOS), make this assumption implicitly. An important aspect of SOS, in common with other models of search (e.g., Humphreys \& Müller, 1993), is the role of similaritybased grouping between display items. According to SOS, search runs through a series of dimension-specific grouping processes, "homing in" on the target. For example, color-based grouping may be used to isolate a candidate target region that may comprise a number of items sharing the target color. This region may then be further segmented into subregions on the basis of a new dimension (e.g., orientation), and so forth, until a target is detected.

5. Despite the fact that the main analyses concerned the speed of the fastest trials (see the RT Distribution Analysis sections), a lower cutoff of $100 \mathrm{msec}$ was used to remove clear anticipatory responses. Since there were fewer false alarm RTs than correct RTs that were less than $100 \mathrm{msec}$, not all correct anticipations would have been removed by the kill-the-twin procedure (Eriksen, 1988; Grice, Nullmeyer, \& Spiker, 1977 ) used prior to the RT distribution analysis.

6. An iterative kill-the-twin procedure was used that eliminated potential fast guesses within $\pm 3 \mathrm{msec}$ of the incorrect-response RT. The procedure searched first for the exact numerical equivalent of the 
incorrect-response RT; if none was found, the procedure searched for an RT within the range $\pm 1 \mathrm{msec}$ of the incorrect-response RT, and so forth up to the maximum range of $\pm 3 \mathrm{msec}$. The twin was not killed when it was outside this range (there were hardly any cases where a twin was not found within the maximum range).

7. The present method of testing for violations of the race model inequality, which was derived directly from the inequality, can be viewed as working vertically, in a typical plot of the CDFs: It compares probabilities of responding by fixed values of time. An alternative method (e.g., Mordkoff \& Yantis, 1991) works horizontally: It compares values of RT at fixed values of probability. Reanalyses of the present data according to this alternative method revealed the same pattern of violations of the race model inequality as the analyses reported above.

8. The data of Experiment 1 do not definitely rule out the possibility that the violations of the race model inequality in the constant-dimension condition were due to lowered response criteria in redundant target blocks. However, the results of Experiment 2 make this an unlikely possibility.

9. Of course, this holds true only if response criteria are not adjusted on a trial-by-trial basis (e.g., lowered after identical repetitions). The present experiments revealed no evidence to this effect. To test for trialby-trial adjustments, negative RTs were compared (1) for target-absent trials following identical-target versus nonidentical-target repetitions and (2) target-absent trials following redundant-target versus nonredundant-target repetitions. None of these comparisons revealed significant differences. This is consistent with the idea that negative response criteria are set in accordance with the average time it takes to detect a target and the variance of the positive responses (rather than the time it took to detect a target on the last trial[s]). There is also other evidence suggesting that response criteria cannot be adjusted rapidly on a trialby-trial basis; rather, they remain largely constant within trial blocks (e.g., Strayer \& Kramer, 1994; Treisman \& Williams, 1984). However, if response criteria were adjusted on a trial-by-trial basis, such adjustments could have affected all previous experiments that have attempted to demonstrate violations of the race model inequality under conditions in which the target-defining dimension(s) varied randomly across trials, such as the experiments of Mordkoff and Yantis (1993). That is, random variation would not effectively guard against criterion effects.

10. We thank Asher Cohen (personal communication, April 3, 2000) for pointing this argument out to us.

11. However, with a single stimulus in the display, the bottom-up saliency signals would not differ between dimensionally redundant and nonredundant targets (or, for that matter, between targets and nontargets). Thus, for redundant targets to produce violations of the race model inequality, top-down processes (e.g., along the lines of GS theory) would have to come into play that raise the saliency for stimuli exhibiting target features in multiple dimensions simultaneously.

(Manuscript received June 11, 1998; revision accepted for publication October 20,2000.) 